\title{
The Norris Survey of the Corona Borealis Supercluster: II. Galaxy Evolution with Redshift and Environment
}

\author{
Todd A. Small ${ }^{1}$ and Wallace L.W. Sargent \\ Palomar Observatory, California Institute of Technology, Pasadena, CA 91125 \\ Electronic mail: tas@ast.cam.ac.uk, wws@astro.caltech.edu \\ Donald Hamilton \\ Max-Planck-Institut für Astronomie, Königstuhl 17, Heidelberg D-69117, Germany \\ Electronic mail: hamilton@mpia-hd.mpg.de
}

\begin{abstract}
We measure the field galaxy luminosity function (LF) as a function of color and redshift from $z=0$ to $z=0.5$ using galaxies from the Norris Survey of the Corona Borealis Supercluster. The data set consists of 603 field galaxies with $0<z \leq 0.5$ and spans a wide range in apparent magnitude $\left(14.0^{m} \lesssim r \lesssim 21.5^{m}\right)$, although our field galaxy LF analysis is limited to 493 galaxies with $r \leq 20.0^{m}$. We use the observed $g-r$ colors of the galaxies to compute accurate corrections to the rest $B_{A B}$ and $r$ bands. We find that our local $r$-band LF, when normalized to counts in high galactic latitude fields, agrees well with the local LF measured in the Las Campanas Redshift Survey. Our $B_{A B}$-band local LF, however, does not match the $b_{j}$-band LF from the Stromlo/APM survey, having a normalization 1.6 times higher. We see compelling evidence that the $B_{A B}$-band field galaxy LF evolves with redshift. The evolution is strongest for the population of star-forming galaxies with [O II] $\lambda 3727$ rest-frame equivalent widths greater than $10 \AA$. The population of red, quiescent galaxies shows no sign of evolution to $z=0.5$. The evolution of the LF which we observe is consistent with the findings of other faint galaxy redshift surveys. The fraction of galaxies with [O II] emission increases rapidly with redshift, but the fraction of galaxies with strong $\mathrm{H} \delta$ absorption, a signature of a burst of star-formation, does not. We thus conclude that the star formation in distant galaxies is primarily long-lived.

We also compute the LFs of the Corona Borealis supercluster $(z \approx 0.07,419$ galaxies with $\left.14.1^{m} \leq r \leq 20.0^{m}\right)$ and the Abell 2069 supercluster $(z \approx 0.11$,
\end{abstract}

\footnotetext{
${ }^{1}$ present address: Institute of Astronomy, University of Cambridge, Madingley Road, Cambridge CB3 OHA, UK
} 
318 galaxies with $\left.15.1^{m} \leq r \leq 20.0^{m}\right)$. The shapes of the two supercluster luminosity functions are broadly similar to the shape of the local luminosity function. However, there are important differences. Both supercluster LFs have an excess of very bright galaxies. In addition, the characteristic magnitude of the Corona Borealis supercluster LF is roughly half a magnitude brighter than that of the local field galaxy LF, and there is a suggestion of an upturn in the LF for galaxies fainter than $M\left(B_{A B}\right) \sim-17^{m}$.

Subject headings: galaxies: evolution-galaxies: distances and redshiftsgalaxies: luminosity function - cosmology: observations

\section{Introduction}

With the introduction of efficient multi-object spectrographs on 4m-class telescopes, it has become possible to construct large samples of faint galaxies with measured redshifts. With such a sample, one can compute the luminosity function (LF) of galaxies as a function of redshift and thereby directly observe the evolution (or lack thereof) of the galaxy population. Several groups have now presented the results of deep, faint galaxy redshift surveys (Lilly et al. 1995, CFRS; Ellis et al. 1996, Autofib; Cowie et al. 1996; Lin et al. 1996a, CNOC). The conclusions from these surveys are in broad agreement: the population of blue, star-forming galaxies has evolved strongly since $z \sim 0.5$ while the population of red galaxies shows at most modest signs of evolution (although, see Kauffmann, Charlot, \& White (1996) for an alternative analysis of the red galaxies). However, there are important differences as well. Lin et al. (1996a) demonstrate that the LFs from the various groups are formally inconsistent with each other. Since there are many selection effects involved with the construction and analysis of faint redshift surveys, it is difficult to pinpoint the reasons for the disagreement between the various groups. While it is likely that the small numbers of galaxies in each survey and the small areas covered are partly responsible, it is also likely that systematic errors are in important contributor to the differences in detail.

Quantitative estimates of the evolution are, of course, dependent upon having a reliable measurement of the local LF, and it is, therefore, of concern that there remain considerable uncertainties about the local LF. The LFs derived from large-area local redshifts survey (e.g., the Stromlo/APM survey, Loveday et al. 1992; the CfA survey, Marzke, Huchra, \& Geller 1994a; the Las Campanas Redshift Survey, Lin et al. 1996b) all have similar shapes, 
but there are still substantial differences over the overall normalization, the characteristic luminosity, and the slope at low luminosities. The rapid evolution at $z \sim 0.1$ required to match steep $B$-band counts at intermediate magnitudes $17^{m}<B<21^{m}$ (Maddox et al. 1990) could be reduced if the normalization or the faint-end slope have been underestimated. The results of the largest of the local surveys, the Las Campanas Redshift Survey (LCRS) with 18678 galaxies used in the LF analysis and a median redshift of $z \approx 0.1$, are seemingly consistent with both a low normalization and a flat faint-end slope. The LCRS is selected from CCD drift scans rather than photographic plates and surveys what should be a fair volume of the universe (Shectman et al. 1996, Davis 1996). It also probes both the Southern and Northern Galactic Caps. Accordingly, the local luminosity function computed from their data should be free from systematic photometric errors and fluctuations in large-scale structure in the distribution of galaxies. However, both the CfA survey and the Autofib survey find a normalization which is a factor of 2 higher than that obtained from the LCRS. While the normalization of the CfA survey can be questioned on the grounds that it does not sample a fair volume, the Autofib survey is the concatenation of many fields distributed across the sky. The Autofib survey is particularly important because the galaxy sample was selected with a much fainter surface brightness threshold than any of the other local surveys. McGaugh (1994) emphasizes that a large population of intrinsically luminous but low surface brightness galaxies may be missed in the shallow photometry on which all the local surveys, except Autofib, are based.

A steep faint-end slope of the LF, with a power law exponent of $\alpha \lesssim-1.5$, is a natural prediction of galaxy formation theories based on hierarchical structure formation models (Kauffmann, Guiderdoni, \& White 1994). There is only weak evidence for a steep faint-end slope in the local field galaxy LF. Marzke et al. (1994b) report an upturn in the luminosity function of late-type galaxies with $M(B)>-16+\log _{10} h$, but LCRS, Autofib, and Cowie et al. (1996) all derive a flat faint-end slope. There is, however, evidence for a steep faint-end slope in galaxy clusters (e.g., De Propris et al. 1995, Bernstein et al. 1995). Environmental influences on galaxy evolution may be reflected in variations of the LF for galaxies in different environments, and it is therefore important to measure the LF in a variety of environments.

In this paper, we investigate the evolution and environmental dependence of the galaxy LF based on data obtained during the course of our redshift survey of the Corona Borealis supercluster. The primary motivation for the survey was to study the dynamics of the supercluster. However, the majority of galaxies for which we measured redshifts actually lie behind the Corona Borealis supercluster, thus providing a sample suitable for study of the evolution of the LF. The galaxies were originally selected from plates taken as part of the Second Palomar Observatory Sky Survey (POSS-II; Reid et al. 1991) and have been 
calibrated in the Gunn $g$ and $r$ bands, which correspond roughly to the photographic $J$ and $F$ bands. Previous redshift surveys have generally been either selected in bluer bands $(B)$, for sensitivity to changes in star-formation rates, or redder bands ( $I$ and $K$ ), for sensitivity to old stellar populations which more reliably trace stellar mass. Although we had no

option but to use the $g$ and $r$ bands, the two bands turn out fortuitously to have the virtue that corrections to the rest $B$ band, where LFs are traditionally computed and compared, are small since the $g$ band matches the rest $B$ band at $z \approx 0.2$ and the $r$ band matches the rest $B$ band at $z \approx 0.5$. The CNOC survey used photometry in $g$ and $r$ as well, and so it is particularly interesting to compare our results to that survey since there should be no systematic effects due to using different passbands for galaxy selection. Finally, with over 400 redshifts in the Corona Borealis supercluster, and roughly 300 in a background supercluster, we can explore the variation of the LF from the field to the supercluster environment.

The paper, the second in the series presenting results from the Norris Survey of the Corona Borealis Supercluster, is organized as follows. In $\S 2$, we summarize our survey, particularly emphasizing those features that are directly relevant to the computation of the LF. We discuss the details of the computation of the LF in $\S 3$. The results are given in $\S 4$ for both field galaxies and for the two superclusters individually and are discussed in $§ 5$. Finally, we summarize our conclusions in $\S 6$.

We use a Hubble constant $H_{0}=100 \mathrm{hm} \mathrm{s}^{-1} \mathrm{Mpc}^{-3}$ and a deceleration parameter $q_{0}=0.5$. For comparison to the most recent work in the field (e.g., CFRS and CNOC), we use the AB-normalized $B$ band, $B_{A B}$ (Oke 1974). The offsets from $B_{A B}$ to $b_{j}$ and $B$ are $b_{j} \approx B_{A B}$ and $B=B_{A B}-0.14^{m}$ (Fukugita, Shimasaku, \& Ichikawa 1995).

\section{The Norris Survey of the Corona Borealis Supercluster}

The Norris Survey of the Corona Borealis Supercluster has been described in detail in Small et al. (1996), Paper I of the current series, and will be only briefly reviewed here. The core of the supercluster covers a $6^{\circ} \times 6^{\circ}$ region of the sky centered at right ascension $15^{h} 20^{m}$, declination $+30^{\circ}$ and consists of 7 rich Abell clusters at $z \approx 0.07$. Since the field-of-view of the 176-fiber Norris Spectrograph is only $400 \mathrm{arcmin}^{2}$, we planned to observe 36 fields arranged in a rectangular grid with a grid spacing of $1^{\circ}$. As it turned out, we successfully observed 23 of the fields and 9 additional fields along the ridge of galaxies between Abell 2061 and Abell 2067, yielding redshifts for 1491 extragalactic objects. We have extended our survey with 163 redshifts from the literature, resulting in 1654 redshifts 
in the entire survey. 1022 of these galaxies lie beyond the Corona Borealis Supercluster, although of these 1022, 325 (318 with $r \leq 20.0^{m}$ ) galaxies are in a background supercluster $(z \approx 0.11)$ which we have dubbed the "Abell 2069 Supercluster." The survey fields are distributed across an area of $25 \mathrm{deg}^{2}$. The total area covered by the 32 observed fields, albeit sparsely sampled, is $2.99 \mathrm{deg}^{2}$.

As noted above and described in detail in Paper I, the objects have been selected from POSS-II photographic plates of POSS-II field 449, which neatly covers the entire core of the supercluster. We have both a $J$ (Kodak III-aJ emulsion with a GG395 filter) plate and an $F$ (Kodak III-aF emulsion with a RG610 filter) plate. The plates were digitized with $1 \operatorname{arcsec}^{2}$ pixels at the Space Telescope Science Institute and then processed using the Sky Image Cataloging and Analysis Tool (SKICAT, Weir 1995). The instrumental intensities recorded by SKICAT were calibrated with CCD sequences in the $g$ and $r$ bands of galaxies in Abell 2069. The random magnitude errors are $0.25^{m}$ for $g$ and $r$ brighter than $21^{m}$ and become substantially worse at fainter magnitudes. (We describe how we correct our computed LFs for these magnitude errors in §3.2.) With the SKICAT system, the star-galaxy separation is $90 \%$ accurate to $r \approx 20^{m}$. Our LF analysis is limited to galaxies with $r \leq 20^{m}$.

Since our original motivation for the survey was to study the dynamics of the Corona Borealis supercluster, we chose a comparatively high spectral resolution for a faint galaxy redshift survey. A third of the objects were observed with $\sim 8 \AA$ spectral resolution during the period when the largest CCD available at Palomar was a $1024^{2}$ device with $24 \mu \mathrm{m}$ pixels; the rest were observed with $\sim 4 \AA$ resolution with a very efficient $2048^{2}$ CCD (also with $24 \mu \mathrm{m}$ pixels). Since the operation of retrieving the fibers for one set-up and redeploying the fibers for another takes roughly an hour with the Norris Spectrograph, we decided to observe only two fields per night in order to minimize the amount of time lost due to changing fields. Thus, our exposures were 2-4 hours long, and we generally obtained high quality spectra on $r \sim 20^{m}$ galaxies.

In Figure 1, we plot our success rate, the fraction of objects (i.e., including stars and quasars) on which fibers were deployed for which we successfully measured redshifts, as a function of magnitude. Figure 1 shows that our success rate falls substantially below unity beyond $r=18.5^{m}$. Therefore, we have computed weights for each galaxy to correct for a our incomplete sampling. The weight for a particular object is defined to be simply the ratio of the total number of objects in the photometric catalog to the number of objects with redshifts in an interval of $0.5^{m}$ centered on the magnitude of the object. This prescription assumes that the redshift distribution of the objects for which we failed to measure redshifts is identical to the redshift distribution of the objects for which we successfully measured redshifts. The color distribution of the objects that we observed but failed to identify is 
similar to that of the objects that we successfully observed, leading us to conclude that we do not suffer biases against particular types of galaxies (Paper I). Moreover, we do not believe that we should have redshift-dependent biases in our success rate. Since we limit the computation of the LF to $z<0.5$, we are unlikely to be affected by a bias in redshift. The $4000 \AA$ break and Ca H, Ca K lines of old stellar populations and the [O II] line of star-forming galaxies are all within our spectral range out to $z=0.5$. We plot the calculated weights as a function of $r$ magnitude for all galaxies with $r \leq 20^{m}$ and which satisfy our surface brightness threshold (see below) in Figure 2. The weights are greater than unity even for bright galaxies because of the sparse sampling of our survey area. (Since the very brightest galaxies $\left(r<15^{m}\right)$ produce scattered light contamination of nearby spectra on the CCD, we usually did not place fibers on galaxies with $r<15^{m}$, and thus the weights increase for the very brightest galaxies.)

In Paper I, we carefully studied the surface brightness selection effects present in our sample. We found that by restricting our sample to objects with $r \leq 20^{m}$ and with core magnitudes $r_{\text {core }} \leq 21.7^{m}$ (where the core magnitude is the integrated magnitude within the central $9 \operatorname{arcsec}^{2}$ ), we are free from surface brightness selection effects. For comparison,

$r_{\text {core }}=21.7^{m}$ corresponds to a central surface brightness of $\mu=24.1 \mathrm{r}$ mag $\operatorname{arcsec}^{-2}$ for a galaxy with an $L^{*}\left(\approx-20.3+5 \log _{10} h\right.$ mag in the $r$ band $)$ disk.

\section{Calculation of the Luminosity Function}

\section{1. $\quad k$-Corrections}

We compute galaxy LFs in the rest-frame $B_{A B}$ band and, for the local LF, in the rest-frame Gunn $r$ band as well. Rest-frame colors and $k$-corrections are computed from the spectral energy distributions compiled by Coleman, Wu, \& Weedman (1980, hereafter CCW). We assign each galaxy a spectral type based on its $g-r$ color and its redshift. Following Lilly et al. (1995), the spectral type is a real number which takes the values 0 for an elliptical galaxy, 2 for an Sbc galaxy, 3 for an Scd galaxy, and 4 for an Im galaxy. We then interpolate between the CCW spectral energy distributions to construct the spectral energy distribution appropriate for the given spectral type. Galaxies whose colors are redder than a CCW E galaxy or bluer than a CCW Im galaxy are simply assigned the spectral energy distribution of an E galaxy or an Im galaxy, respectively. The number of galaxies with colors outside the limits defined by the CCW E and Im types is known to be small even to large redshifts (e.g., Crampton et al. 1995). The fact that many of our 
galaxies lie outside the CCW limits (see Figure 17, Paper I) is due to the large errors in our colors $\left(\sigma_{g-r} \approx 0.35^{m}\right)$.

We compute the absolute rest-frame $B_{A B}$-band magnitude as follows:

$$
\begin{aligned}
M\left(B_{A B}\right)_{\mathrm{rest}} & =M(r)_{\mathrm{rest}}+\left(B_{A B}-r\right)_{\mathrm{rest}} \\
& =r_{\mathrm{obs}}-5 \log _{10} D_{L}(z)-25 .-k_{r}(z)+\left(B_{A B}-r\right)_{\mathrm{rest}} \\
& =r_{\mathrm{obs}}-5 \log _{10} D_{L}(z)-25 .+2.5 \log _{10}(1+z)-k_{\text {eff }}
\end{aligned}
$$

where $k_{\text {eff }}$ incorporates the corrections based on the spectral energy distribution and $D_{L}$ is the luminosity distance in Mpc. The $2.5 \log _{10}(1+z)$ term represents the change in the bandwidth with redshift and is included in the traditional $k$-correction. Again following Lilly et al. (1995), we separate the bandwidth stretching term, which has negligible error since it depends only on the accurately measured redshift, from the terms which depend on the spectral energy distribution and are therefore much more uncertain. We plot $k_{e f f}$ for the $g$ and $r$ bands in Figure 3. By converting from $g_{\text {obs }}$ for objects with $z \lesssim 0.3$ and from $r_{\text {obs }}$ for objects with $z \gtrsim 0.3, k_{\text {eff }}$ may be kept less than $\pm 0.6^{m}$ for $z<0.7$ for all spectral types.

\subsection{Method}

We use the step-wise maximum-likelihood (SWML) method of Efstathiou, Ellis, \& Peterson (1988) to estimate the LF. The probability of observing a galaxy of absolute magnitude $M_{i}$ at redshift $z_{i}$ in a flux-limited catalog is given by,

$$
p_{i} \propto \frac{\phi\left(M_{i}\right)}{\int_{-\infty}^{M_{\max }\left(z_{i}\right)} \phi(M) d M},
$$

where $\phi$ is the LF and $M_{\max }\left(z_{i}\right)$ is the intrinsically faintest galaxy observable at $z_{i}$ in the flux-limited catalog. The LF is parameterized as a set of $N_{p}$ numbers $\phi_{k}$ such that

$$
\phi(M)=\phi_{k}, \quad M_{k}-\Delta M / 2<M<M_{k}+\Delta M / 2, \quad k=1, \ldots, N_{p},
$$

and then the likelihood,

$$
\mathcal{L}=\prod_{i=1}^{N} p_{i}
$$


where $N$ is the number of galaxies in the sample, is maximized with respect to the $\phi_{k}$. We constrain the values of $\phi_{k}$ to satisfy

$$
\sum_{k=1}^{N_{p}} \phi_{k} \Delta M=1 .
$$

The virtues of the SWML method are that it is not biased by the presence of clustering since the normalization of the LF cancels out of the expression for the probability $p_{i}$ and also that one does not have to assume a particular functional form for the LF. In order to include the weights, we make the substitution

$$
\mathcal{L}=\prod_{i=1}^{N} p_{i} \rightarrow \mathcal{L}=\prod_{i=1}^{N} p_{i}{ }^{w_{i}},
$$

where $w_{i}$ is the weight of galaxy $i$ (Zucca, Pozzetti, \& Zamorani 1994, Lin et al. 1996b). One must then estimate the mean galaxy density separately. We use a standard technique which we describe below. Since the weights are greater than one, their use will increase the calculated likelihood for the sample and thus lead to artificially small error estimates. By renormalizing the weights so that $\sum_{i=1}^{N} w_{i}=N$, the error estimates are appropriate for the true sample size (Lin et al. 1996b). Note that the normalization constraint on the $\phi_{k}$ reduces the estimated errors.

We do not use the traditional $1 / V_{\max }$ method (Schmidt 1968) employed by Ellis et al. (1996) and Lilly et al. (1995) since the method is sensitive to clustering. We have, however, compared the results of the two techniques for samples with $z>0.2$, where the clustering in our survey is not pronounced, and found that they agree satisfactorily. For $z<0.2$, we can construct volume-limited sub-samples with $r \leq 20.0^{m}$ in which any galaxy with $M\left(B_{A B}\right) \leq-19^{m}+5 \log _{10} h$ is visible in the entire volume. Of course, the value of the LF in a given magnitude bin for a volume-limited sample is estimated by counting the number galaxies with absolute magnitudes in the bin and then dividing by the volume of the sample and the width of the bin. The SWML LFs for $z<0.2$ agree well with the LFs estimated from the volume-limited samples.

We compute the mean density $\bar{n}$ of a magnitude-limited sub-sample using the following estimator:

$$
\bar{n}=\frac{1}{V} \sum_{i=1}^{N} \frac{w_{i}}{s\left(z_{i}\right)},
$$

where $V$ is the volume of the sample, $N$ is the number of objects in the sample, and $s$ is the selection function. The selection function,

$$
s\left(z_{i}\right)=\frac{\int_{-\infty}^{M_{l i m}(z)} \phi(M) d M}{\int_{-\infty}^{M_{\max }} \phi(M) d M},
$$


gives the fraction of the LF observable at a given redshift. Here, $\phi(M)$ is the LF, $M_{\text {lim }}(z)$ is the maximum absolute magnitude that an object can have at redshift $z$ and still be included in the sample, and $M_{\max }$ is the absolute magnitude of the most instrinsically faint galaxy in the sample. In practice, one does not begin evaluating the integrals at $-\infty$, but rather at the absolute magnitude of the most instrinsically bright galaxy in the sample. The estimator in Equation 8 is almost identical to the minimum variance estimator derived by Davis \& Huchra (1982) for $s \gtrsim 0.1$ and is unbiased by density inhomogeneities.

An additional complication of computing a LF in the $B_{A B}$ band where the objects have been selected in the $r$ band is that one must ensure that any object, regardless of its color, would have been detectable in both bands. If one ignores this complication, then the faintest objects at a given redshift will be biased in color. In our survey, since the $r$ band is centered at a longer wavelength than the $B$ band, the faintest objects would be biased to the red. In order to avoid such a bias, we adjust our absolute $B_{A B}$ magnitude limits as a function of redshift so that the bluest galaxy at any $B_{A B}$ magnitude limit would be observable in the $r$ band. For the local LF computed in the $r$ band, the bias works in the opposite sense, and so we adjust our rest-frame absolute $r$ magnitude limits to ensure that the reddest galaxy a given limit would be detected in the observed $r$ band.

For each LF, we estimate the parameters of the best-fitting Schechter (1976) function,

$$
\phi(M) d M=\phi^{*} e^{-e^{.92\left(M^{*}-M\right)}+.92\left(M^{*}-M\right) \alpha},
$$

where $\phi^{*}$ is the normalization, $M^{*}$ determines the location of the bright-end exponential cutoff, and $\alpha$ is the faint-end slope. The fitting was performed using a standard $\chi^{2}$ minimization algorithm (Press et al. 1992) with the Schechter function integrated over the width of the adopted magnitude bin. We intend these fits to be useful for comparisons with other work. Usually, there are too few points for the fits to be well defined.

Our random magnitude errors $\left(\sigma \approx 0.25^{m}\right)$ will artificially brighten the characteristic magnitude $M^{*}$ of the LF and steepen the faint end. We correct for the magnitude errors by fitting to the data points a Schechter function (Equation 9) convolved with a Gaussian of dispersion $0.25^{m}$ (Efstathiou et al. 1988). In fact, however, the corrections to the Schechter function parameters are substantially less than the $1 \sigma$ statistical errors.

The error in the normalization from the $\chi^{2}$-fitting only includes the uncertainties due to $M^{*}$ and $\alpha$. The error due to large-scale structure fluctuations is

$$
\frac{\delta \bar{n}}{\bar{n}} \sim\left(\frac{J_{3}}{V}\right)^{1 / 2},
$$

(Davis \& Huchra 1982), where $J_{3}$ is the second moment of the two-point spatial correlation function (Peebles 1980) and $V$ is the appropriate volume. We use $J_{3} \approx 10,000\left(h^{-1} \mathrm{Mpc}\right)^{3}$ 
(Tucker et al. 1997) and record the error from density fluctuations alongside the uncertainty in $\phi^{*}$ due to $M^{*}$ and $\alpha$.

\section{Results}

In the following subsections, we report our results for the local LF, the evolution of the LF, and the LFs of the Corona Borealis and Abell 2069 superclusters. All samples are magnitude-limited at $r=20^{m}$. The parameters of the best fitting Schechter functions are summarized in Table 1, where the sample is given in the first column, the number of galaxies used to compute the LF in the second column, the absolute range over which the fit is valid in the third column, $\phi^{*}$ in the fourth column, $M^{*}$ in the fifth column, $\alpha$ in the sixth column, the reduced $\chi^{2}$ in the seventh column, and the estimate of the variance due to density fluctuations in the eighth column. The numbers of galaxies listed in the second column are slightly smaller than the total number of galaxies satisfying the sample listed in the first column because a few galaxies have been trimmed from each sample, as described above, to ensure that there are no color biases in the faintest bins. Corrections to $\phi^{*}$ to match galaxy counts, as discussed below, have not been applied to the values listed in Table 1. We wish to emphasize that the fitted Schechter functions are intended only to guide the eye and that comparisons of the various LFs in this paper are best done by comparing the individual data points in the plots.

\subsection{The Local Luminosity Function}

The $B_{A B}$-band local galaxy LF is plotted in Figure 1 . The unfilled circles show the LF for $z \leq 0.2$ with the superclusters removed. The filled circles show the LF for $z \leq 0.2$ with the the superclusters included. In order to remove the superclusters, we simply delete all objects with $0.06 \leq z \leq 0.13$. The median redshift of our local sample with the superclusters removed is $z_{\text {med }}=0.15$. The Stromlo/APM LF is plotted with the solid line. We also plot the Autofid local LF with the dashed line. In Figure 1 and all subsequent figures where appropriate, we convolve the Schechter function fits to the LFs from other surveys with a Gaussian of dispersion $0.25^{\mathrm{m}}$ in order to facilitate comparisons with our LF data points, which are constructed with galaxies whose photometry suffers from random magnitude errors of $0.25^{m}$. All of the local luminosity functions have similar shapes, but the normalizations and low luminosity ends vary significantly. 
In order to investigate further the normalization of the local luminosity function, we compute the shape of local luminosity in the rest-frame $r$ band and then normalize this LF to the $r$-band counts of Weir, Djorgovski, \& Fayyad (1995). We plot our $r$-band local LF, normalized to the counts of Weir et al. (1995), in Figure 5, along with the $r$-band local LF from LCRS. To convert from isophotal $R_{L C R S}$ magnitudes to total Gunn $r$ magnitudes, we apply a $25 \%$ isophotal-to-total light correction and then use $R_{L C R S, t o t a l}-r \approx 0.25^{\mathrm{m}}$ (Shectman et al. 1996). Thus, the corrections compensate for each other and $R_{L C R S} \approx r$. The counts of Weir et al. (1995) are based on 4 overlapping, high galactic latitude plates taken as part of the POSS-II survey. Knowing the shape of the LF, we can estimate the differential number counts as

$$
\begin{aligned}
\frac{d n}{d m} & =\int_{0}^{\infty} \phi[M(m, z)] \frac{d V}{d z} d z \\
& =\phi^{*} \int_{0}^{\infty} \phi^{\prime}[M(m, z)] \frac{d V}{d z} d z \\
& =\phi^{*} \frac{d I}{d m}
\end{aligned}
$$

where $\phi^{\prime}$ is $\phi$ with $\phi^{*}$ set equal to $1, M(m, z)$ is the absolute magnitude of an object at redshift $z$ with apparent magnitude $m$, and the $m_{i}$ are the magnitude intervals. We estimate $\phi^{*}$ by minimizing the quantity

$$
\sum_{i} \frac{\left[d n\left(m_{i}\right)-\phi^{*} d I\left(m_{i}\right)\right]^{2}}{\phi^{*} d I\left(m_{i}\right)}
$$

with respect to $\phi^{*}$ (Efstathiou et al. 1988), which yields

$$
\left(\phi^{*}\right)^{2}=\frac{\sum_{i}\left[d n\left(m_{i}\right)\right]^{2} / d I\left(m_{i}\right)}{\sum_{i} d I\left(m_{i}\right)} .
$$

We find $\phi^{*}=1.5_{-0.5}^{+0.7} \times 10^{-2} h^{3} \mathrm{Mpc}^{-3}$ where the errors reflect the changes due to varying jointly $M^{*}$ and $\alpha$ by $\pm 1 \sigma$. This is a $21 \%$ reduction from the normalization determined in the Norris field itself. Although the median redshift of our local sample is $z_{\text {med }}=0.15$, the agreement with LCRS $\left(z_{\text {med }} \approx 0.1\right)$ in the $r$ band leads us to believe that we have computed a fair estimate of the local LF.

In Figure 6, we plot the $r$-band differential number counts. The histogram shows the counts from the POSS-II plate from which we selected our objects. The thick solid line is the counts from Weir et al. (1995), and the triangles are CCD counts from Metcalfe et al. (1991). The dashed line represents the predicted counts based on our field galaxy LF, including evolution at $z>0.2$ (see $\S 4.2$ below), with the superclusters removed. Even with the superclusters removed, our local LF appears to be normalized too high. The solid 
line, however, gives the predicted counts with the normalization reduced by $21 \%$. With the reduced normalization, the predicted counts agree quite well with the observed counts to $r=20^{m}$. For comparison, we also show, as the dotted line, the predicted counts from the LCRS. These counts fall below the Weir et al. (1995) counts for $r \geq 18^{m}$ because they do not include the evolution of the LF beyond $z \geq 0.2$. Since the volume of the Norris region, with the superclusters removed, is $\approx 2.6 \times 10^{5} h^{-3} \mathrm{Mpc}^{3}$, we would expect from equation 10 $\delta \bar{n} / \bar{n} \approx 0.20$. It is therefore not cause for concern that this region is overdense by $21 \%$.

\subsection{The Luminosity Function to $z=0.5$}

We compute the field galaxy LF in two redshift intervals: $0<z<0.2$ and $0.2<z<0.5$. The results are plotted in Figure 0 . The normalization of the local LF (unfilled circles) has been reduced by $21 \%$ to match the $r$-band counts. The filled circles are the LF of the high redshift interval. We also plot the LFs of the Stromlo/APM survey (solid line), the CNOC survey $(0.2<z<0.6$, dashed line), and the CFRS survey ( $0.2<z<0.5$, dotted line), all of which have been convolved with a Gaussian with $\sigma_{m}=0.25^{m}$ to account for random photometry errors. Since the LF for the Autofib survey was divided into redshift intervals which do not neatly match our redshift intervals and, more importantly, since Lin et al. (1996a) have already performed a detailed comparison with the Autofib survey, we do not plot the Autofib LFs. The $0.2<z<0.5 \mathrm{LF}$ has clearly evolved with respect to the local LF.

\subsection{The Luminosity Function to $z=0.5$ Divided by $W_{0}([\mathrm{O} \mathrm{II}])$}

We create two sub-samples of galaxies according to the rest-frame equivalent width of [O II] $\lambda 3727$. The division is made at a rest-frame equivalent width of $10 \AA$, which roughly corresponds to dividing the sample into types earlier and later than Sbc (Kennicutt 1992) and thus allows direct comparison with the results of CNOC and CFRS. For galaxies with $z<0.049$, [O II] is not redshifted into our observed wavelength range, and so we use the strength of $\mathrm{H} \beta$ to divide our sample. After correcting for stellar absorption, we have 7 galaxies with $z<0.049$ and $W_{0}(\mathrm{H} \beta)>5 \AA$, which we include in the $W_{0}([\mathrm{O}$ II $])>10 \AA$ sample. We have also investigated separating our sample by color, but we have found that the large errors on our colors tend to dilute trends which are seen clearly in samples defined 
by $W_{0}([\mathrm{O} \mathrm{II}])$. The LFs for galaxies with $W_{0}([\mathrm{O} \mathrm{II}])<10 \AA$ and $W_{0}([\mathrm{O} \mathrm{II}])>10 \AA$ are shown in Figure 8 and Figure 9, respectively. We also plot in each figure the LFs for the corresponding color-selected samples from CNOC and CFRS. It is important to remember when considering possible detailed discrepancies between our LFs and the CNOC and CFRS LFs that our sample is divided by $W_{0}\left(\left[\begin{array}{ll}\mathrm{O} & \mathrm{II}\end{array}\right]\right)$, which, while roughly equivalent to color selection, is not identical. There is no significant indication that the population of early-type galaxies (i.e., $\left.W_{0}([\mathrm{O} \mathrm{II}])<10 \AA\right)$ has evolved since $z=0.5$; this result is not surprising given that the light of early-type galaxies is dominated by red, long-lived stellar populations. In contrast, the LFs of the late-type galaxies (i.e., those with $W_{0}([\mathrm{O} \mathrm{II}])>$ $10 \AA)$ show striking evidence for evolution, even though the sample sizes are small and the error bars are large.

\subsection{The Supercluster Luminosity Functions}

The LFs of the Corona Borealis supercluster and the Abell 2069 supercluster are given in Figure 10. We take the redshift range of the Corona Borealis supercluster to be $0.06 \leq z \leq 0.09$ and that of the Abell 2069 supercluster to be $0.10 \leq z \leq 0.13$. The normalization of the Corona Borealis supercluster function is a factor of 2 greater than that of the Abell 2069 supercluster, but the shapes of the LFs of the two superclusters are similar. Note that the volumes used to normalize the superclusters LFs are in redshift space; the real-space volumes may be quite different (see $\S 5.3$ below for a detailed discussion). Since the bright ends of the supercluster LFs are clearly not well-described by a Schechter function, we restrict our fit to $M\left(B_{A B}\right)>-21.3^{m}$. In addition, the fit to the Corona Borealis supercluster is limited to $M\left(B_{A B}\right)<-17.1^{m}$ since the faintest two data points appear to describe a sharp upturn in the LF.

\section{Discussion}

\subsection{The Local Luminosity Function}

Prior evidence for rapid evolution of the galaxy LF to $z \sim 0.1$ from galaxy counts was based crucially on normalizing the local LF to the bright $\left(B_{A B} \sim 16^{m}\right)$ galaxy counts from Schmidt-telescope photographic surveys (e.g., Maddox et al. 1990). With this low 
normalization, predicted counts from the no-evolution model fall well short of the observed counts for $B_{A B}>18^{m}$. However, the evolution of the LF which we and others observe is not enough to make up for this shortfall. In Figure 11, we plot the observed counts from the APM survey (Maddox et al. 1990) and from the CCD survey of Metcalfe et al. (1991), along with various predicted counts. The dotted line shows the expected counts using the Loveday et al. (1992) LF for $0<z<0.2$ and the CNOC $B_{A B}$ LF for $z>0.2$. Despite including the observed evolution of the LF, the predicted counts only begin to agree with the observed counts for $B_{A B} \gtrsim 19^{m}$, by which point the predicted counts are dominated by galaxies with $z>0.2$. In contrast, the predicted counts computed using the evolving LFs measured for our survey and for the Autofib survey, while substantially overpredicting the counts for $B_{A B} \lesssim 18^{m}$, match the observed counts for $18^{m} \lesssim B_{A B} \lesssim 20.5^{m}$.

In order to help unravel this confusing situation, we plot in Figure 12 various $B_{A B^{-}}$ and $r$-band local LFs on the same diagram. $B_{A B}$-band LFs are plotted with respect to the bottom axis, while $r$-band LFs are plotted with respect to the top axis. The two axes are offset by $B_{A B}-r=0.72^{m}$, which is the median color we measure for field galaxies with $z<0.2$. Our $B_{A B^{-}}$and $r$-band local LFs, both of which have been reduced by $21 \%$ following the discussion in $\S 4.1$, are plotted with filled and unfilled circles, respectively. With the color offset, they agree extremely well. The Stromlo/APM LF, represented by the solid line, lies consistently below our $B_{A B}$-band LF. The LCRS $r$-band LF is consistent with our $r$-band LF. Unlike Lin et al. (1996b), we do not conclude that the LCRS $r$-band LF matches the Stromlo/APM LF. The reason for the disagreement lies in the different measurements of the median color of local galaxies. Our median color is that of an $\mathrm{Sb}$ galaxy, whereas the LCRS median color is that of a much redder E galaxy. The median color of galaxies in the Third Reference Catalogue of Bright Galaxies (de Vaucouleurs et al. 1991 ) is $\langle B-V\rangle \approx 0.75^{m}$ (see Table 2 of Fukugita et al. 1995), which is roughly that of an Sb galaxy. Sebok (1986) also concludes that the typical local galaxy has the color of an Sb galaxy. It is thus surprising that the mean color of the LCRS galaxies is so red. Since our $r$-band LF agrees with the LCRS $r$-band LF and since the LCRS computed the colors of their galaxies by matching directly to the APM catalog, the most natural explanation for the anomalous red colors of the LCRS is a systematic error in the bright APM magnitudes. We note that Weir et al. (1995) conclude that magnitudes derived from $J$ plates are only reliable for $g>16^{m}$ or, equivalently, $B_{A B}>16.5^{m}$. Galaxies brighter than $B_{A B} \approx 16.5^{m}$ are saturated on the photographic plates. A systematic error in the bright APM counts would remove the need for rapid, and otherwise unsubstantiated, galaxy evolution at $z \sim 0.1$.

We note also that there is possible evidence for a rise in the local LF above an $\alpha \approx-1$ for the least luminous galaxies in our survey. Such a rise is evident in the data of Marzke et al. (1994b) for irregular galaxies, the CFRS, and the low surface brightness galaxy 
redshift survey of Sprayberry et al. (1997). Similar behavior is also perhaps visible in the local LF of the Autofib survey. Although Ellis et al. (1996) argue against a rise in the LF for $M\left(B_{A B}\right)>-16^{m}$, the three faintest points in the their local LF (their Figure 8) all lie above their preferred Schechter function fit. However, since such faint galaxies are only visible in our survey in a quite small volume, we refrain from attempting to make a definitive statement.

\subsection{The Evolution of the Luminosity Function to $z=0.5$}

We asserted in $\$ 4.2$ that the LF of star-forming galaxies $\left(W_{0}([\mathrm{O} \mathrm{II}])>10 \AA\right)$ evolved from $z=0$ to $z=0.5$ and that the luminosity function of galaxies with weak [O II] emission $(<10 \AA)$ did not. A powerful method to verify this result is to compute $\left\langle V / V_{\max }\right\rangle$ for appropriate samples (Schmidt 1968). If there is no evolution in the number density of objects, $\left\langle V / V_{\max }\right\rangle=0.5$; if the number density declines, $\left\langle V / V_{\max }\right\rangle<0.5$; and if the number density increases, $\left\langle V / V_{\max }\right\rangle>0.5$. Our $\left\langle V / V_{\max }\right\rangle$ analysis is complicated by the need to remove the superclusters and to account for the $21 \%$ overdensity of our local field. In order to excise the superclusters, we simply remove all galaxies with $0.06 \leq z \leq 0.13$. If the maximum redshift $z_{\max }$ at which a galaxy could be observed in our survey lies in the range 0.06 to 0.13 , we set $z_{\max }=0.06$. We correct for the overdensity of our local field by reducing the weights of galaxies with $z \leq 0.2$ by $21 \%$. The values of $\left\langle V / V_{\max }\right\rangle$ for various samples of galaxies are given Table 2 , both with and without the $21 \%$ correction to the weights of the galaxies with $z \leq 0.2$. For samples selected by color, we compute the $\left\langle V / V_{\text {max }}\right\rangle$ statistic for galaxies in the redshift range $0<z \leq 0.5$ (with the supercluster region excluded). For samples selected by the strength of [O II], we use the redshift range $0.049<z \leq 0.5$ (with the supercluster region excluded) since [O II] from objects with $z<0.049$ is not redshifted into our observed wavelength range. The differences between our weighted and unweighted statistics are small. The $\left\langle V / V_{\max }\right\rangle$ test supports our claim, at the $3.5 \sigma$ level for the weighted statistic, that the population of star-forming galaxies is evolving. The rate of evolution increases with the strength of $[\mathrm{O} \mathrm{II}]$. The population of galaxies with $W_{0}([\mathrm{O} \mathrm{II}])$ $>20 \AA$ has $\left\langle V / V_{\max }\right\rangle=0.65 \pm 0.04$. This result is analogous to the results from CFRS in which the rate of evolution is the strongest for the bluest population of galaxies. $\left\langle V / V_{\max }\right\rangle$ for the population of red galaxies is consistent with no evolution, in agreement with the LF analysis.

Broadly speaking, our results are in accord with the results of CFRS, Autofib, Cowie et al (1996), and CNOC. Since the CNOC survey, like our survey, uses photometry in the 
$g$ and $r$ bands, it is particularly interesting to compare our results in detail to theirs since many of the systematic effects associated with $k$ - and color-corrections ought to be the same. It is encouraging to see (Figure 8 and 9) that, given the small samples, our LFs agree well with those of CNOC. The agreement is significantly improved if one reduces the normalization of the CNOC LFs by $20 \%$, as, in fact, is recommended by Lin et al. (1996a).

Now that we have confirmed that the population of blue galaxies is evolving with redshift, we wish to investigate whether we can detect differences in the colors and the spectral properties of the evolving population with redshift. First, we reiterate that the color distribution of objects with measured redshifts is similar to the color distribution of unidentified objects, leading us to believe that the type distribution of the identified objects is not strongly biased (Paper I). Although emission lines are generally easier to detect than absorption lines, the difficulty of identifying emission lines at observed wavelengths longer than $5577 \AA$, where there are many strong night sky features, combined with the strength of $\mathrm{Ca} \mathrm{H}, \mathrm{Ca} \mathrm{K}$, and the $4000 \AA$ break in absorption line objects at $0.4 \lesssim z \lesssim 0.6$ mitigate the bias in favor of emission line objects. A sample of the spectra of 8 absorption line objects in this redshift range is shown in Figure 13 to illustrate the ease of detection of their characteristic absorption features.

In Figure 14, we plot the observed $g-r$ color as a function of redshift of all the objects in our survey along with the tracks of five representative model galaxy spectra. The bluest spectrum is simply a flat-spectrum object, $f_{\nu}=0$. The four other spectra are typical of the Hubble types E, Sbc, Scd, and Im and are taken from CCW. The large, solid diamonds mark the observed median color in the redshift ranges $0.13<z<0.2$ (arranged to exclude the superclusters), $0.2<z<0.3,0.3<z<0.4,0.4<z<0.5$, and $0.5<z<0.6$. Perhaps counter-intuitively, we see that the observed median color does not become progressively bluer with respect to the model spectra with increasing redshift. As discussed by Lilly et al. (1995) and illustrated in our Figure 15, the median color does not become bluer because the color-magnitude relation in the local universe (i.e., the fact that more luminous galaxies are redder) breaks down for $z \gtrsim 0.2$ as the population of blue galaxies brightens while the population of red galaxies does not evolve significantly. We plot in Figure 15 the absolute $B_{A B}$-band magnitudes versus rest-frame $g-r$ for our galaxies divided into four intervals in redshift. In the low-redshift interval, the color-magnitude relation is apparent, but it disappears in the higher redshift intervals. At $z \lesssim 0.2$, we observe far down the LF to absolute magnitudes where blue galaxies are dominant. At $z \gtrsim 0.2$, we do not observe as far down the LF, but the population of blue galaxies has brightened so as to be included in the samples.

The increase in the luminosity of the population of blue galaxies is presumably 
associated with a change in the star formation activity at earlier times. In our spectra, there are two convenient star formation indicators, [O II] $\lambda 3727$ and $\mathrm{H} \delta \lambda 4101$. [O II] emission is found in galaxies with ongoing star formation, and its strength is proportional to the strength of $\mathrm{H} \alpha$ (Kennicutt 1992). Strong $\mathrm{H} \delta$ absorption is a signature of the presence of a population of A-stars, which are visible $\sim 1$ Gyr after a burst of star formation. These lines are reliably measured by automated programs (see Paper I) since both occur in regions of the spectrum where the continuum is featureless and there is little crowding from other lines. An important virtue of the $\mathrm{H} \delta$ line is that, since it appears in absorption, a galaxy with detectable $\mathrm{H} \delta$ would have been identified no matter what its spectral characteristics, which implies that there is no bias towards detecting objects with $\mathrm{H} \delta$ absorption. A galaxy with [O II] emission is, of course, easier to identify than if it had had only absorption lines. However, as we discussed above, the combination of the difficulty of identifying weak emission lines in the face of strong sky subtraction residuals and of the ease of identifying the strong features characteristic of absorption-line galaxies at moderate redshifts suggests that our survey is not strongly biased towards detecting objects with [O II]. By adding together spectra of late-type galaxies in two redshift intervals in order to obtain two spectra with very high signal-to-noise ratios, Heyl et al. (1996) find that the two star-formation indicators [O II] $\lambda 3727$ and $\mathrm{H} \delta$ have both become stronger in the higher redshift mean spectrum. Those authors interpreted the increase in the strength of [O II] and $\mathrm{H} \delta$ in the $z>0.2$ spectra to imply that not only were the higher-redshift galaxies forming stars more rapidly, but that also the nature of the star formation was changing with redshift. Specifically, they asserted that the strong $\mathrm{H} \delta$ absorption in the higher redshift sample is evidence that the star formation in that sample is dominated by bursts. With our high-quality spectra, we can reliably measure [O II] and $\mathrm{H} \delta$ without adding together the spectra of many galaxies. In Figure 16, we plot as a function of redshift the fraction of galaxies with $[\mathrm{O} \mathrm{II}]$ emission and the fraction of galaxies with $\mathrm{H} \delta$ absorption. The vertical dashed line marks the redshift beyond which $\mathrm{H} \delta$ is shifted into the region of the spectrum in which sky subtraction becomes increasingly difficult. While the fraction of galaxies with [O II] emission increases with redshift, the fraction of galaxies with strong $\mathrm{H} \delta$ absorption shows no significant variation with redshift, suggesting that the star formation is not occurring in short-lived bursts (c.f., Hammer et al. 1997)

In order to investigate this disagreement more carefully, we repeat the analysis of Heyl et al. (1996) and construct high signal-to-noise ratio composite spectra. The individual spectra have been median-combined after scaling by the median count level. The magnitude weights, which correct for our incompleteness at faint magnitudes, have not been applied since scaling by the median count level in each spectrum effectively incorporates the magnitude weights. In Figure [17, we plot the composite spectra of galaxies with 
$W_{0}([\mathrm{O} \mathrm{II}])>20 \AA, M\left(B_{A B}\right) \leq-19^{m}$, and either $0.13<z \leq 0.2$ (thick line, $z_{\text {med }}=0.16$ ) or $0.2<z \leq 0.5$ (thin line, $\left.z_{\text {med }}=0.38\right)$. $W_{0}([\mathrm{O}$ II $])>20 \AA$ is typical for the late-type galaxies in which Heyl et al. (1996) observe spectral changes with redshift. While the [O II] line is modestly stronger in the higher redshift composite spectrum, there is no difference in the strength of $\mathrm{H} \delta$ (which is partially filled-in by emission in both spectra), which indicates that the nature of the star formation has not changed from $z=0.5$ to $z=0$. In contrast to Heyl et al. (1996), we again do not conclude that the spectra of intermediate-redshift blue galaxies show spectral signs of short bursts of star formation. (We do not believe that the small differences in the rest-equivalent widths of the $\mathrm{Ca} \mathrm{H}$ and $\mathrm{K}$ lines are significant since it is difficult to fit a reliable continuum in the vicinity of the $4000 \AA$ break.)

The modest increase in the strength of the [O II] line with redshift for galaxies with $M_{B_{A B}} \leq-19^{m}$ is due, as previously discussed by Cowie et al. (1996), to the increase in the intrinsic luminosity of star-forming galaxies with redshift. In Figure 18, we plot absolute magnitude versus the rest equivalent width of [O II] for the galaxies in our sample divided into four redshift intervals. As the redshift increases, more and more luminous galaxies exhibit strong [O II]. Notice, however, that the range of rest equivalent widths does not vary significantly with redshift.

It is difficult to draw firm conclusions about the nature of the evolving galaxy population from the LFs alone. The LFs provide only a statistical view of the entire population and include information about the evolution of individual galaxies only indirectly. Furthermore, the luminosity of a galaxy is an unreliable tracer of the physical state of the galaxy. The luminosity of a galaxy, especially in the rest-frame ultraviolet, can change dramatically on short timescales, making the identification of the descendants of distant galaxies in the local population very difficult. In order to make further progress, additional data on the nature of distant galaxies is required. The morphologies of distant galaxies, measured with the Hubble Space Telescope (HST), are already providing crucial clues (Driver et al. 1995, Glazebrook et al. 1995b, Abraham et al. 1996). The counts of faint elliptical and early-type spiral galaxies match predictions based on counts in the local universe, provided that the local LF is normalized, as advocated here, a factor of 1.5-2 higher than found by Loveday et al. (1992). In contrast, the HST number counts of late-type and irregular galaxies are far in excess of the counts expected from observations of nearby galaxies, even with a high normalization of the local LF.

An alternative method for selecting distant galaxies is by their gas absorption cross section. Steidel, Dickinson, \& Persson (1994) study the evolution of 58 galaxies selected by Mg II $\lambda \lambda 2796,2803$ absorption seen in the spectra of high-redshift quasars. These authors found that the galaxies responsible for quasar absorption lines in the range $0.2 \leq z \leq 1.0$, 
which typically have luminosities near $L^{*}$, do not evolve over the redshift range. However, their sample is small and, when divided by color, is not inconsistent with results from surveys of galaxies selected by apparent magnitude (Lilly et al. 1995). In addition, Steidel et al. (1994) note that intrinsically faint blue galaxies do not appear in their sample, and so the population of galaxies which is observed to be evolving most rapidly is not included in their survey.

It is now possible, especially with $10 \mathrm{~m}$-class telescopes, to measure the masses (e.g., Vogt et al. 1993, 1996; Rix et al. 1996, Guzmán et al. 1996) and chemical abundances of distant galaxies. Since the masses and metallicities of galaxies evolve more smoothly than the luminosities, measurements of these two quantities will allow more easily interpretable comparisons of distant and local populations (Guzmán et al. 1996). In addition, the mass and metallicity are, compared to morphology and even gas absorption cross section (Churchill, Steidel, \& Vogt 1996), straightforward to define and interpret. A survey to measure the masses and chemical abundances of the faint blue galaxies ought to yield important insights into the nature of this rapidly evolution galaxy population and aid in the identification of their present-day counterparts.

\subsection{The Supercluster Luminosity Functions}

The LFs of the two superclusters have quite similar shapes and generally resemble the field galaxy LF. Since the normalizations of the superclusters LFs are computed in redshift space, in which distances along the line-of-sight may be substantially altered with respect to real space, it is not straighforward to compare the normalization of the supercluster LFs with that of the local field galaxy LF. For the purpose of the discussion here, we introduce a factor $f$, the ratio of the redshift-space volume to the real-space volume. We expect $f$ to be in the range $1 \lesssim f \lesssim 5$. The lower limit corresponds to assuming that the peculiar velocities in the supercluster regions are small; the upper limit corresponds to assuming that the depth of the superclusters along the line-of-sight is similar to the linear sizes of the superclusters on the plane of the sky $\left(\sim 20 h^{-1} \mathrm{Mpc}\right)$. The real-space mean density of galaxies in the Corona Borealis supercluster, obtained simply by integrating the measured LF data points $\left(-22.1^{m} \leq M\left(B_{A B}\right) \leq-16.3^{m}\right)$, is $\bar{n}_{C B} \approx 0.4 f_{C B}^{-1} h^{3} \mathrm{Mpc}^{-3}$. The mean density of field galaxies in the same range of absolute magnitude is $\bar{n}_{\text {field }} \approx 0.05 h^{3}$ $\mathrm{Mpc}^{-3}$. Similarly, the real-space mean density of galaxies in the A2069 supercluster $\left(-21.7^{m} \leq M\left(B_{A B}\right) \leq-17.5^{m}\right)$ is $\bar{n}_{A 2069} \approx 0.1 f_{A 2069}^{-1} h^{3} \mathrm{Mpc}^{-3}$, while the mean density of field galaxies in the same range of absolute magnitude is $\bar{n}_{\text {field }} \approx 0.04 h^{3} \mathrm{Mpc}^{-3}$. Thus, the 
overdensities are $\sim 40 f_{C B}^{-1}$ and $\sim 13 f_{A 2069}^{-1}$ for the Corona Borealis and A2069 superclusters, respectively.

It is also important to know whether our sampling of the superclusters is biased towards either the Abell clusters within the superclusters or the "field" of the superclusters. Owing to the difficulties of converting redshift-space volumes to real-space volumes, we assess our sampling of the superclusters using projected surface densities, which are not affected by redshift-space distortions. For the Corona Borealis supercluster, the project surface density $\Sigma_{C B}$ is the mean redshift-space galaxy volume density multiplied by the depth in redshift space of the supercluster along the line-of-sight, $c \Delta z / H_{0}=90 h^{-1}$ Mpc. Thus, $\Sigma_{C B} \approx 0.4 \times 90 h^{2} \mathrm{Mpc}^{-2}=36 h^{2} \mathrm{Mpc}^{-2}$. We compare this value with the median surface density of the regions surrounding successfully observed Corona Borealis supercluster galaxies. We compute the surface density around a given Corona Borealis supercluster galaxy by counting the number of supercluster galaxies in a 6 arcmin diameter circle surrounding the chosen galaxy. We either simply count the number of galaxies with measured redshifts within the Corona Borealis supercluster, or we count the total number of galaxies on the original POSS-II $F$ plate, weighted by the empirically-determined fraction of galaxies at a given magnitude which are in the supercluster. The results of these two methods agree well: the median values of the raw and weighted surface densities are $\Sigma_{\text {raw,med }}=27 h^{2} \mathrm{Mpc}^{-2}$ and $\Sigma_{\text {weighted,med }}=42 h^{2} \mathrm{Mpc}^{-2}$. These two values bracket the projected surface density measured by multiplying the mean galaxy density (computed from the LF) by the line-of-sight depth of the supercluster. We conclude, therefore, that we have fairly sampled the Corona Borealis supercluster.

We perform an identical analysis for the background A2069 supercluster. The redshift-space depth of the A2069 supercluster is also $90 h^{-1}$ Mpc. Given a mean galaxy volume density of $\bar{n}_{A 2069} \approx 0.1 h^{3} \mathrm{Mpc}^{-3}$, the projected surface density is $\Sigma_{A 2069}=9 h^{2}$ $\mathrm{Mpc}^{-2}$. The median surface density of the regions surrounding the successfully observed A2069 supercluster galaxies, computed in the same fashion as for the Corona Borealis supercluster, is $\Sigma_{\text {med,raw }}=13 h^{2} \mathrm{Mpc}^{-2}$ (unweighted) or $\Sigma_{\text {med,weighted }}=16 h^{2} \mathrm{Mpc}^{-2}$ (weighted). Thus, we are slightly biased to the denser regions of the A2069 supercluster.

The overall resemblance between the supercluster LFs and the field galaxy LF suggests that the fundamental physical processes which drive galaxy formation and evolution must not depend strongly on environment. There are, however, important differences between the LFs in the field and in the superclusters that must ultimately be due to environmental effects. The most striking difference between the field and supercluster LFs is that the supercluster LFs to do not continue the exponential decline for galaxies brighter than $M\left(B_{A B}\right) \lesssim-21^{m}$. Both superclusters evidently contain a population of very luminous 
galaxies. Despite the fact that there are only 6 galaxies with $M\left(B_{A B}\right)<-21.3^{m}$ in the two superclusters combined, it is clear that these galaxies are giant ellipticals found in the densest regions of the superclusters. The 4 of the 6 for which we have spectra (the other 2 were taken from the literature) are dominated by the light of an old, red stellar population. All of the galaxies are found in regions in the upper 27th percentile of local surace density, with half of them found in regions in the upper 10th percentile. In fact, the four galaxies in the Corona Borealis supercluster are all found in the dense ridge of galaxies between the Abell clusters A2061 and A2067. For the Corona Borealis supercluster, the characteristic magnitude $M^{*}$ is $\sim 0.5^{m}$ brighter than in the field and is quite close to the value measured by Colless (1989) for rich clusters. There is no significant difference between $M^{*}$ for the Abell 2069 supercluster and that of the field.

For the Corona Borealis supercluster LF, the data points for the two faintest magnitude bins hint that the LF may steepen significantly for galaxies fainter than $M\left(B_{A B}\right) z-17^{m}$. Since the hint is based on only two data points, which are themselves based on only 29 galaxies, we must be cautious in our interpretation. However, a steepening of the supercluster LF fainter than $M\left(B_{A B}\right) \gtrsim-17^{m}$ would be in accord with observations of the faint end of the LF in galaxy clusters and groups, in which a number of workers report steep ( $\alpha \lesssim-1.3$ ) LFs (Impey, Bothun \& Malin 1988; Ferguson \& Sandage 1991; Biviano et al. 1995; De Propris et al. 1995; Driver \& Phillipps 1996). With the exception of the study of the Coma cluster by Biviano et al. (1995), the observations of steep faint ends in cluster and group LFs have depended, since redshifts were not available, on the subtraction of a background component from the foreground cluster, a procedure which is prone to systematic errors. Although it would be unwise to draw strong conclusions from our two data points, they do have the virtue of being based on galaxies with measured redshifts.

\section{Summary}

We have presented an analysis of the LF of galaxies in the Norris Survey of the Corona Borealis Supercluster. Our $r$-band LF of local field galaxies, when normalized to counts in high galactic latitude fields, agrees well with the LCRS. However, the normalization of our $B_{A B}$ local LF is roughly a factor of 1.6 higher than that of the Stromlo/APM survey. Since Lin et al. (1996b) claim that the LCRS local LF agrees well with the Stromlo/APM survey, the difference must lie in a systematic photometry error in one (or more) of the three surveys. A clue to the nature of this error is provided by examining the mean colors of Norris and LCRS galaxies. The mean color of local Norris galaxies is that of an Sb galaxy, 
whereas the mean color of LCRS galaxies, computed by matching LCRS galaxies directly with galaxies in the APM catalog, is that of an E galaxy. Given the agreement of the Norris and LCRS $r$-band LFs, we therefore believe that the error is most likely in the APM catalog. Indeed, brightening the magnitudes of APM galaxies with $b_{j}<17^{m}$ by $\sim 0.25^{m}$ would bring all of the local LFs into agreement. A CCD-based local redshift survey (e.g., the Sloan survey, Gunn \& Weinberg 1995) will certainly resolve any remaining questions about the local LF.

We have observed evolution of the field galaxy LF within our sample, thereby confirming the conclusions drawn from several previous redshift surveys. The evolution is limited to the population of blue, star-forming galaxies. The population of blue galaxies becomes more luminous with increasing redshift, and thus the median color of the field galaxy population does not change. The evolution of the population of blue galaxies is reflected in the larger fraction of galaxies at higher redshift exhibiting spectral signatures of ongoing star formation. In contrast to the results of Heyl et al. (1996), but in agreement with Hammer et al. (1996), we find that the star formation is long-lived. We do not see evidence for short-term bursts of star formation. We are unable to detect any evolution of the population of galaxies with $W_{0}([\mathrm{O}$ II $])<10 \AA$. The fact that the evolution which we observe in our $g$ - and $r$-band selected survey is consistent with the results of the surveys of Lilly et al. (1995), Ellis et al. (1996), Cowie et al. (1996), and Lin et al. (1996) adds to the already strong evidence that a consistent picture of the evolution of the galaxy LF is emerging. In particular, it is quite reassuring that our LFs agree well with those of Lin et al. (1996a) since both used the $g$ and $r$ bands and should therefore have very similar systematic effects.

The LFs of the two superclusters show significant differences from the field galaxy LF, despite considerable overall similarity. Since the superclusters are $\sim 10-40 \times$ denser than the field, we are likely to be observing the influence of the environment on galaxy formation and evolution. The most prominent difference is an excess of very bright galaxies $\left(M\left(B_{A B}\right) \lesssim-21^{m}\right)$ relative to the best-fitting Schechter function, which accurately describes the field LF over the observed absolute magnitude range. These very bright galaxies are found in very dense regions of the superclusters and have spectra dominated by an old, red stellar population. In the Corona Borealis supercluster, the characteristic magnitude $M^{*}$ is $\sim 0.5^{m}$ brighter than in the field. $M^{*}$ for the Abell 2069 supercluster is, however, very close to the value in the field. We have also presented suggestive evidence that there is a sharp upturn in the supercluster LF for $M\left(B_{A B}\right) \gtrsim-17^{m}$. While there is also a suggestion of an upturn in the local field galaxy LF for the least luminous galaxies in our survey, it does not appear as dramatic as the upturn seen in the supercluster LF, but more data are needed before the possible difference can be quantified. 
We are grateful to the Kenneth T. and Eileen L. Norris Foundation for their generous grant for construction of the Norris Spectrograph. We thank the staff of the Palomar Observatory for the expert assistance we have received during the course of the survey, David Hogg for many enlightening discussions, and the referee for a careful reading of this paper and helpful suggestions. This work has been supported by an NSF Graduate Fellowship (TAS) and NSF grant AST-92213165 (WLWS). 
TABLE 1

Convolved Schechter Function Fits

\begin{tabular}{|c|c|c|c|c|c|c|c|}
\hline sample ${ }^{a}$ & $N$ & Mag. range ${ }^{\mathrm{b}}$ & $\begin{array}{c}\phi^{*} \\
\left(h^{3} \mathrm{Mpc}^{-3}\right)\end{array}$ & $M^{*}-5 \log _{10} h$ & $\alpha$ & $\chi^{2} / \nu^{c}$ & $\left(\frac{J_{3}}{V}\right)^{1 / 2}$ \\
\hline \multicolumn{8}{|c|}{$B_{A B}$} \\
\hline$z \leq 0.2, \mathrm{SC}^{\prime}$ s removed & 219 & $-21.3--15.2$ & $0.028 \pm 0.010$ & $-19.51 \pm 0.27$ & $-1.02 \pm 0.18$ & $5.9 / 9$ & 0.20 \\
\hline$z \leq 0.2$, SC's included & 940 & $-22.1--15.2$ & $0.028 \pm 0.075$ & $-20.02 \pm 0.19$ & $-1.21 \pm 0.10$ & $13.3 / 11$ & 0.17 \\
\hline $0 . \overline{2}<z \leq 0.5$ & 210 & $-21.7--19.0$ & $0.041 \pm 0.006$ & $-19.12 \pm 0.20$ & $-0.24 \pm 0.51$ & $3.2 / 4$ & 0.06 \\
\hline$W_{0}([\mathrm{O} \mathrm{II}])<10 \AA, z \leq 0.2, \mathrm{SC}$ 's removed & 159 & $-21.3--16.1$ & $0.011 \pm 0.006$ & $-19.97 \pm 0.46$ & $-1.09 \pm 0.23$ & $9.6 / 3$ & 0.20 \\
\hline$W_{0}([\mathrm{O} \mathrm{II}])>10 \AA, z \leq 0.2, \mathrm{SC}$ 's removed & 60 & $-20.4--15.2$ & $0.011 \pm 0.014$ & $-19.26 \pm 1.08$ & $-1.10 \pm 0.45$ & $0.4 / 2$ & 0.20 \\
\hline$W_{0}([\mathrm{O} \mathrm{II}])<10 \AA, 0.2<z \leq 0.5$ & 157 & $-21.7--19.2$ & $0.022 \pm 0.004$ & $-19.44 \pm 0.29$ & $-0.79 \pm 0.60$ & $6.3 / 7$ & 0.06 \\
\hline$W_{0}([\mathrm{O} \mathrm{II}])>10 \AA, 0.2<z \leq 0.5$ & 53 & $-21.3--19.0$ & $0.033 \pm 0.013$ & $-19.10 \pm 0.58$ & $-0.87 \pm 1.28$ & $0.7 / 1$ & 0.07 \\
\hline Corona Borealis SC & 404 & $-21.3--17.1$ & $0.091 \pm 0.032$ & $-20.04 \pm 0.34$ & $-1.10 \pm 0.15$ & $11.8 / 8$ & 0.60 \\
\hline Abell 2069 SC & 284 & $-21.3--17.5$ & $0.079 \pm 0.024$ & $-19.56 \pm 0.26$ & $-1.06 \pm 0.18$ & $7.5 / 6$ & 0.42 \\
\hline \multicolumn{8}{|c|}{$r$} \\
\hline$z \leq 0.2$, SC's removed & 236 & $-22.0--15.5$ & $0.019 \pm 0.008$ & $-20.45 \pm 0.30$ & $-1.04 \pm 0.17$ & $6.2 / 12$ & 0.20 \\
\hline
\end{tabular}

aSC stands for "supercluster."

b Absolute magnitude range over which fits are valid.

${ }^{c} \nu$ is the number of degrees of freedom of the fit. 
TABLE 2

$\left\langle V / V_{\max }\right\rangle$ FOR $0<z \leq 0.5$

\begin{tabular}{lccc}
\hline \hline \multicolumn{1}{c}{ sample } & $\begin{array}{c}\left\langle V / V_{\max }\right\rangle \\
\text { unweighted }\end{array}$ & $\begin{array}{c}\left\langle V / V_{\max }\right\rangle \\
\text { weighted }\end{array}$ & Number of Galaxies \\
\hline$r \leq 20.0$, Redder than Sbc & $0.51 \pm 0.02$ & $0.53 \pm 0.02$ & 354 \\
$r \leq 20.0, \mathrm{Bluer}$ than $\mathrm{Sbc}$ & $0.54 \pm 0.02$ & $0.55 \pm 0.02$ & 139 \\
$r \leq 20.0, \mathrm{~W}([\mathrm{O}$ II $])<5 \AA$ & $0.48 \pm 0.02$ & $0.49 \pm 0.02$ & 284 \\
$r \leq 20.0, \mathrm{~W}([\mathrm{O} \mathrm{II}])>5 \AA$ & $0.57 \pm 0.02$ & $0.58 \pm 0.02$ & 193 \\
$r \leq 20.0, \mathrm{~W}([\mathrm{O} \mathrm{II}])>10 \AA$ & $0.57 \pm 0.03$ & $0.59 \pm 0.03$ & 47 \\
$r \leq 20.0, \mathrm{~W}([\mathrm{O} \mathrm{II}])>20 \AA$ & $0.63 \pm 0.04$ & $0.65 \pm 0.04$ & 47 \\
\hline
\end{tabular}




\section{REFERENCES}

Abraham, R., Tanvir, N., Santiago, B., Ellis, R., Glazebrook, K., \& van den Bergh, S. 1996, MNRAS, 279, L47

Bernstein, G., Nichol, R., Tyson, J., Ulmer, M., \& Wittman, D. 1995, AJ, 110, 1507

Biviano, A., Durret, F., Gerbal, D., Le Fèvre, O., Lobo, C., Mazure, A., \& Slezak, E. 1995, A\&A, 297, 610

Bruzual, G. \& Charlot, S. 1993, ApJ, 405, 538

Churchill, C., Steidel, C., \& Vogt, S. 1996, ApJ, 471, 164

Coleman, G., Wu, C.-C., \& Weedman, D. 1980, ApJS, 43, 393

Colless, M. 1989, MNRAS, 237, 799

Cowie, L., Songaila, A., Hu, E., \& Cohen, J. 1996, AJ, 112, 839

Crampton, D., Le Fèvre, O., Lilly, S., \& Hammer, F. 1995, ApJ, 455, 96

Davis, M. 1996, preprint astro-ph/9610149

Davis, M. \& Huchra, J. 1982, ApJ, 254, 437

De Propris, R., Pritchet, C., Harris, W., \& McClure, R. 1995, ApJ, 450, 534

De Vaucouleurs, G., De Vaucouleurs, A., Corwin, H., Buta, R., Paturel, G., \& Fouqué, P. 1991, Third Reference Catalogue of Bright Galaxies (New York: Springer)

Driver, S., Windhorst, R., Ostrander, E., Keel, W., Griffiths, R., \& Ratnatunga, K. 1995, ApJ, 449, L23

Driver, S., \& Phillipps, S. 1996, ApJ, 469, 529

Efstathiou, G., Ellis, R., \& Peterson, B. 1988, MNRAS, 232, 431

Ellis, R., Colless, M., Broadhurst, T., Heyl, J., \& Glazebrook, K. 1996, MNRAS, 280, 235

Ferguson, H., \& Sandage, A. 1991, AJ, 101, 765

Fukugita, M., Shimasaku, K., \& Ichikawa, T. 1995, PASP, 107, 945

Glazebrook, K., Ellis, R., Santiago, B., \& Griffiths, R. 1995b, MNRAS, 275, L19 
Gunn, J., \& Weinberg, D. 1995 in Wide-Field Spectroscopy, ed. S. Maddox \& A. Aragón-Salamanca (Singapore: World Scientific), p. 3

Guzman, R., Koo, D., Faber, S., Illingworth, G., Takamiya, M., Kron, R., \& Bershady, M. 1996, ApJ, 460, L5

Hammer, F., Flores, H., Lilly, S., Crampton, D., Le Fèvre, O., Rola, C., Mallen-Ornelas, G., Schade, D., \& Tresse, L. 1996, preprint astro-ph/9612071

Heyl, J., Colless, M., Ellis, R., \& Broadhurst, T. 1996, MNRAS, in press

Impey, C., Bothun, G., \& Malin, D. 1988, ApJ, 330, 634

Kauffmann, G., Guiderdoni, B., \& White, S. 1994, MNRAS, 267, 981

Kauffmann, G., Charlot, S., \& White, S. 1996, MNRAS, 283, L117

Kennicutt, R. 1992, ApJ, 388, 310

Lilly, S., Tresse, L., Hammer, F., Crampton, D., \& Le Fèvre, O. 1995, ApJ, 455, 108

Lin, H., Yee, H., Carlberg, R., \& Ellingson, E. 1996a, ApJ, 475, 494

Lin, H., Kirshner, R., Shectman, S., Landy, S., Oemler, A., Tucker, D., \& Schechter, P. 1996b, ApJ, 464, 60

Loveday, J., Peterson, B., Efstathiou, G., \& Maddox, S. 1992, ApJ, 390, 338

Maddox, S., Sutherland, W., Efstathiou, G., Loveday, J., \& Peterson, B. 1990, MNRAS, $247,1 \mathrm{P}$

Marzke, R., Huchra, J., \& Geller, M. 1994a, ApJ, 428, 43

Marzke, R., Geller, M., Huchra, J., \& Corwin, H. 1994b, AJ, 108, 437

McGaugh, S. 1994, Nature, 367, 538

Metcalfe, N., Shanks, T., Fong, R., \& Jones, L. 1991, MNRAS, 249, 493

Metcalfe, N., Shanks, T., Fong, R., \& Roche, N. 1995, MNRAS, 273, 257

Metcalfe, N., Fong, R., \& Shanks, T. 1995, MNRAS, 274, 769

Oke, J. 1974, ApJS, 27, 21 
Peebles, P. 1980, The Large-Scale Structure of the Universe (Princeton: Princeton University Press)

Press, W., Teukolsky, S., Vetterling, W., \& Flannery, B. 1992, Numerical Recipes 2nd. edn. (Cambridge: Cambridge University Press)

Reid, I. et al. 1991, PASP, 331, 465

Rix, H., Guhathakurta, P., Colless, M., \& Ing, K. 1996, preprint, astro-ph/9605204

Schechter, P. 1976, ApJ, 203, 297

Shectman, S., Landy, S., Oemler, A., Tucker, D., Lin, H., Kirshner, R., \& Schechter, P. 1996, ApJ, 470, 172

Schmidt, M. 1968, ApJ, 151, 393

Sebok, W. 1986, ApJS, 62, 301

Small, T., Sargent, W., \& Hamilton, D. 1996, ApJS, in press (Paper I)

Sprayberry, D., Impey, C., Irwin, M., \& Bothun, G. 1997, ApJ, in press astro-ph/9701051

Steidel, C., Dickinson, M., \& Persson, S. 1994, ApJ, 437, L75

Tucker, D., Oemler, A., Kirshner, R., Lin, H., Shectman, S., Landy, S., Schechter, P., Müller, V., Gottlöber, S., \& Einasto, J. 1996, preprint astro-ph/9611206.

Vogt, N., Herter, T., Haynes, M., \& Courteau, S. 1993, ApJ, 415, 95L

Vogt, N., Forbes, D., Phillips, A., Gronwall, C., Faber, S., Illingworth, G., \& Koo, D. 1996, ApJ, 465, L15

Weir, N. 1995, Ph.D. thesis, California Institute of Technology

Weir, N., Djorgovski, S., \& Fayyad, U. 1995, AJ, 110, 1

Zucca, E., Pozzetti, L., \& Zamorani, G. 1994, MNRAS, 269, 953 


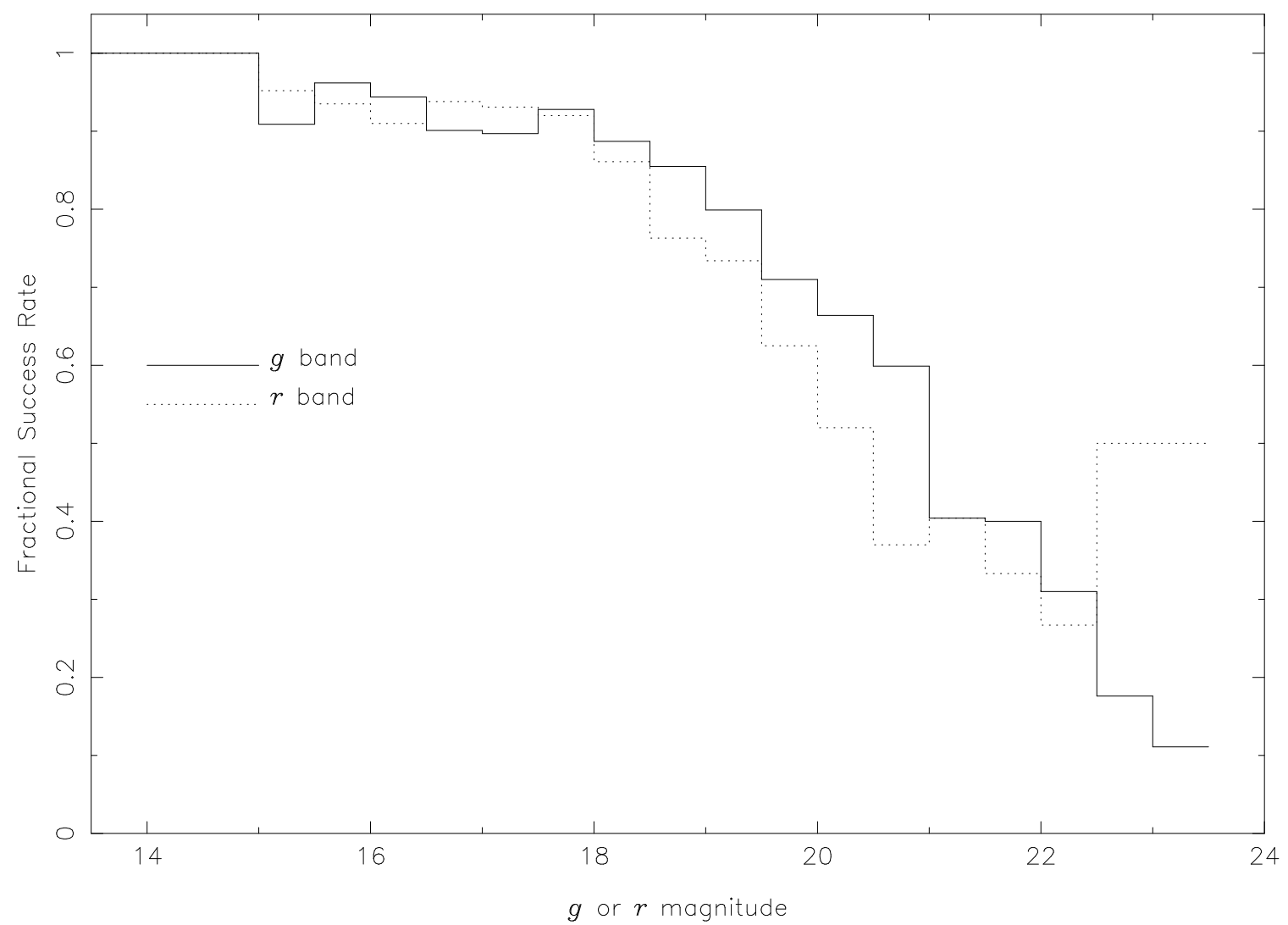

Fig. 1.- Redshift identification success rate as a function of $g$ (solid line) and $r$ (dotted line) magnitudes. 


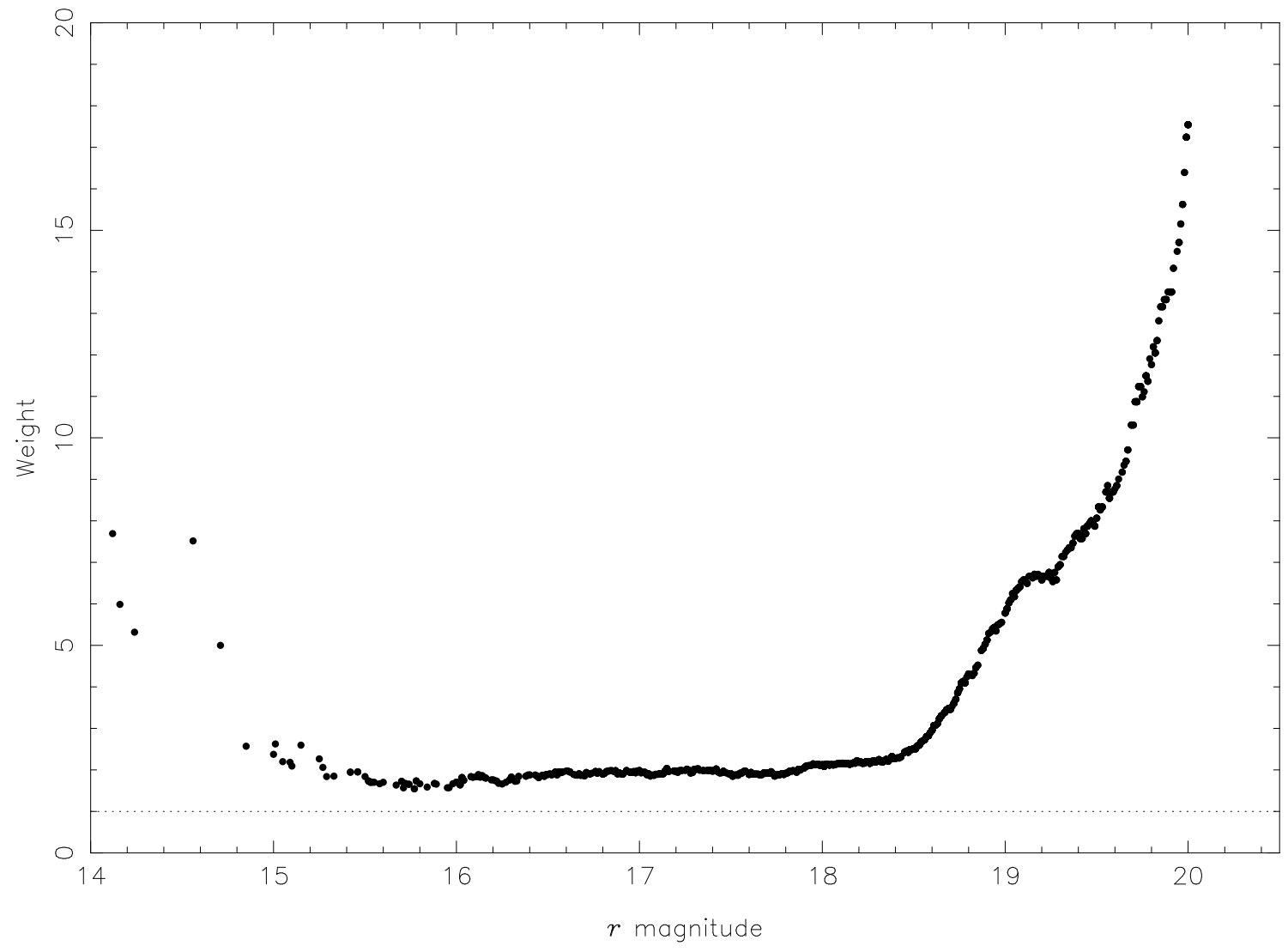

Fig. 2.- Selection weights as a function of $r$ magnitude. The weights, even for bright galaxies, do not reach unity because we sparsely sampled our survey area. Since scattered light from the very brightest galaxies with $r \leq 15^{m}$ would contaminate adjacent spectra on the CCD, we purposely avoided observing such galaxies, and thus the weights actually increase for $r \leq 15^{m}$. 


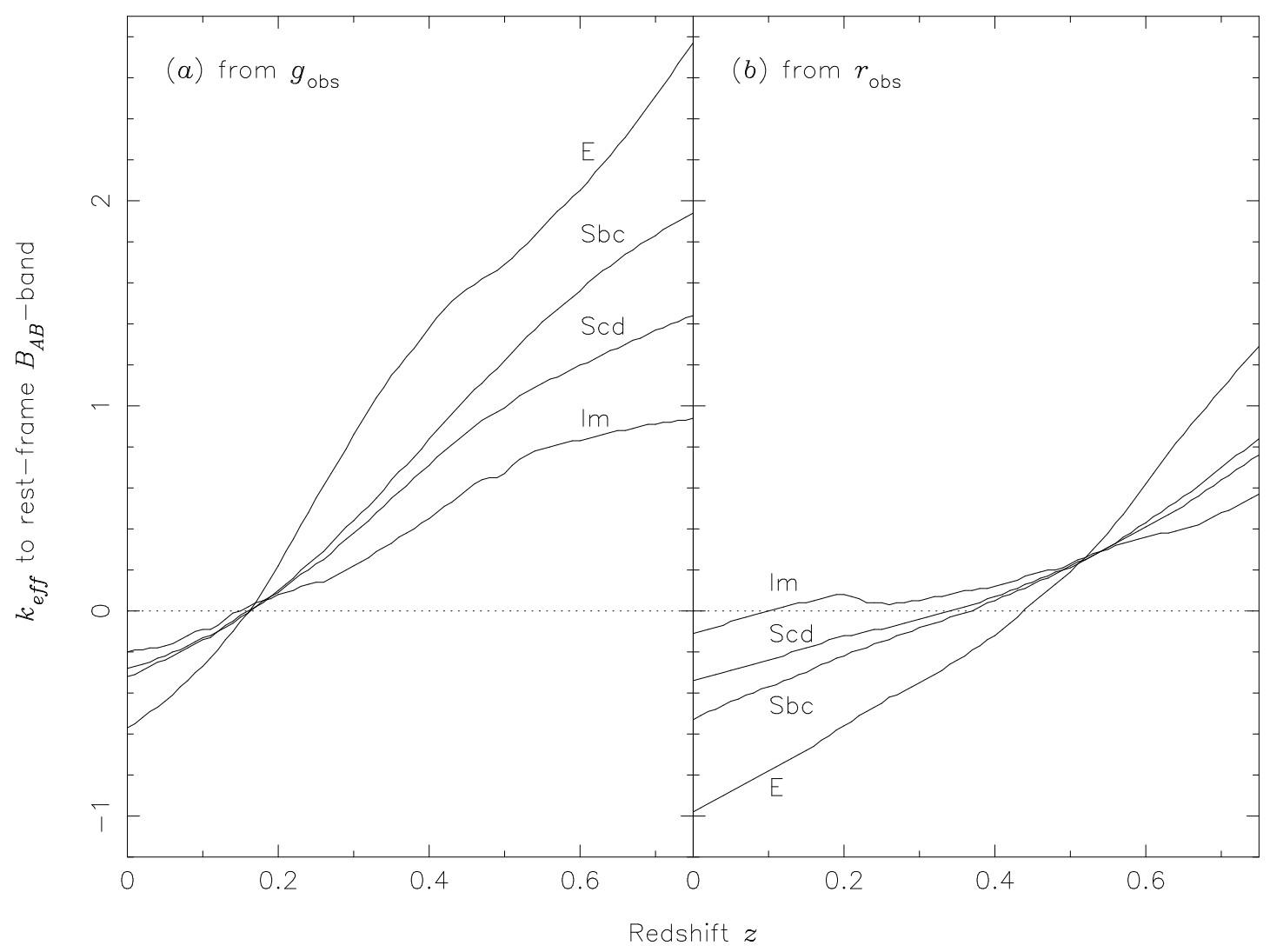

Fig. 3.- Effective $k$-corrections from the observed $(a) g$ and $(b) r$ bands to the rest-frame $B_{A B}$ band. The effective $k$-correction, $k_{e f f}$, is the traditional $k$-correction with the bandwidth stretching term removed and the rest-frame color correction to the $B_{A B}$ band added. It thus incorporates all of the spectrum-dependent corrections required to transform from the observed band to the rest-frame $B_{A B}$ band. The four curves in each panel labeled E, Sbc, Scd, and Im are the four spectral types from CCW. By transforming from the observed $g$ band for $z \lesssim 0.3$ and from the observed $r$ band for $0.3 \lesssim z \lesssim 0.5$, the correction is always less than $\pm 0.6^{m}$. 


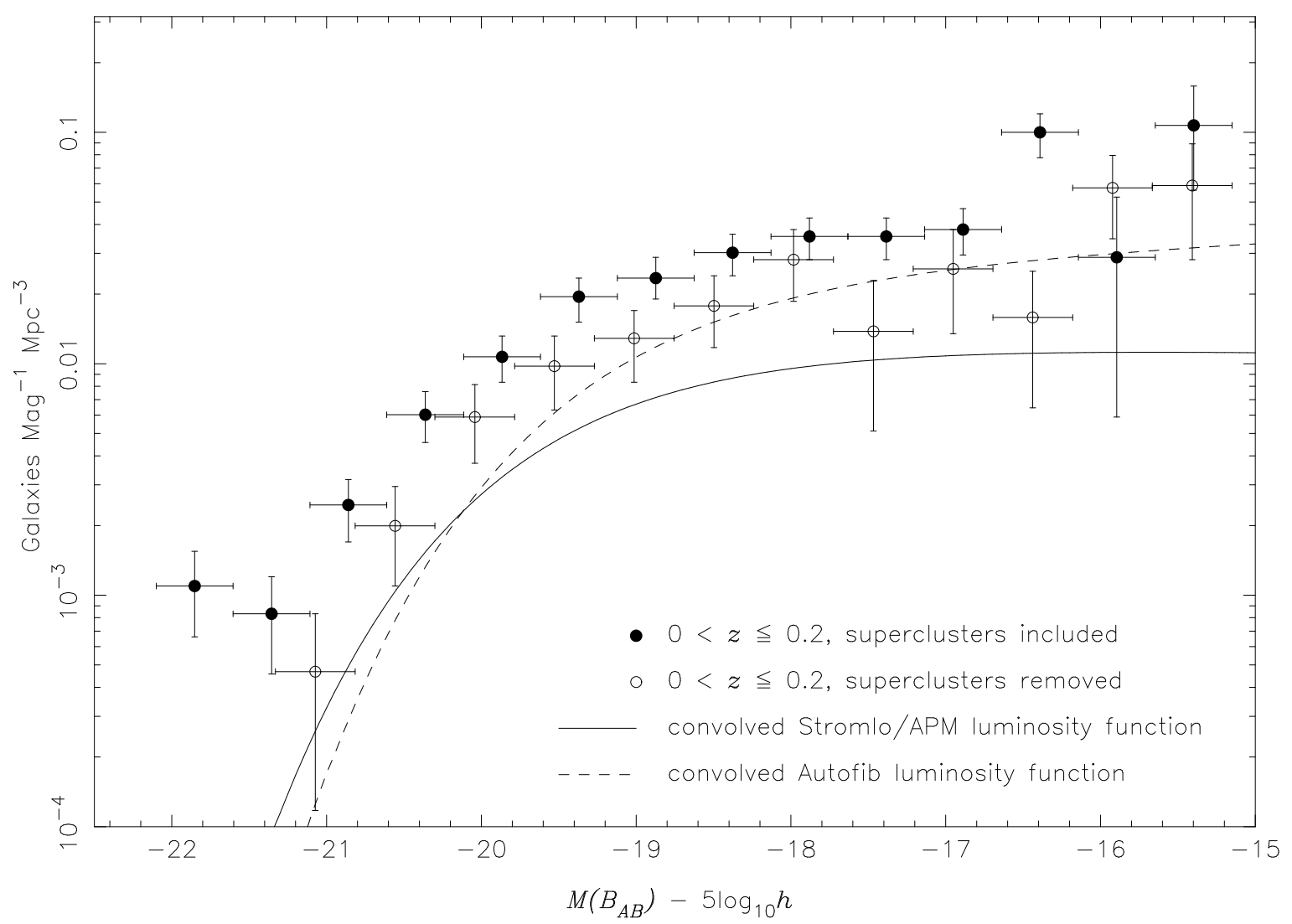

Fig. 4. - $B_{A B}$-band LF for galaxies with $0<z \leq 0.2$. The unfilled circles show the LF with the two superclusters at $z \approx 0.07$ and $z \approx 0.11$ removed. The filled circles show the LF with the superclusters included. The solid and dashed lines give the Schechter function fits to the Stromlo/APM and Autofib local LFs, respectively, convolved with a Gaussian of dispersion $0.25^{m}$ to account the random magnitude errors in our survey. (When reproducing the LFs of other surveys in subsequent figures, we will always convolve the published LFs with a Gaussian of dispersion $0.25^{m}$.) While all the $B_{A B}$-band local LF's have similar shapes, there are substantial differences in the normalization. 


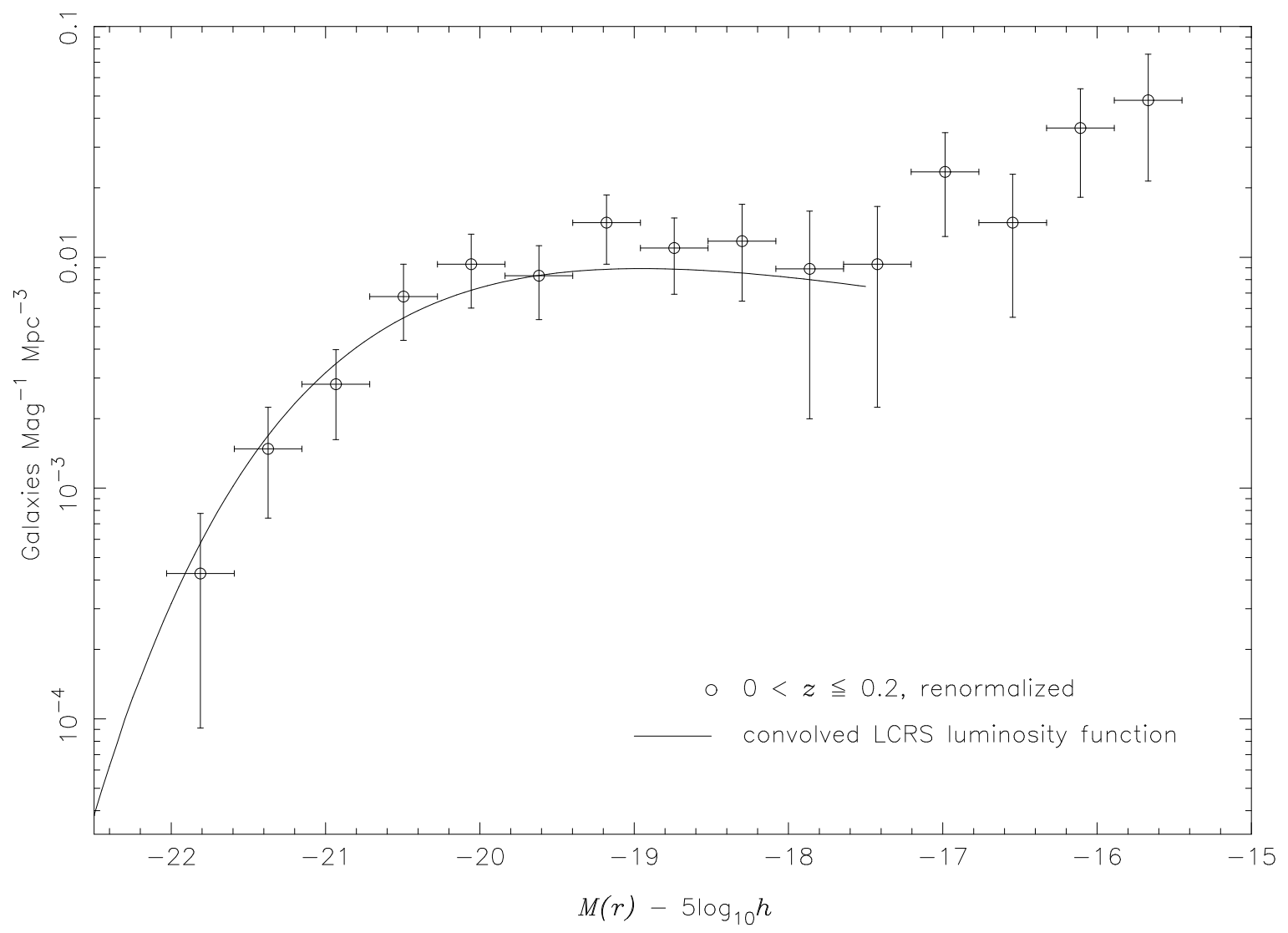

Fig. 5.- $r$-band LF for galaxies with $0<z \leq 0.2$. The unfilled circles show our $r$-band local LF, normalized to the counts of Weir et al. (1995). The solid line gives Schechter function fit to the local LF measured in the LCRS. 


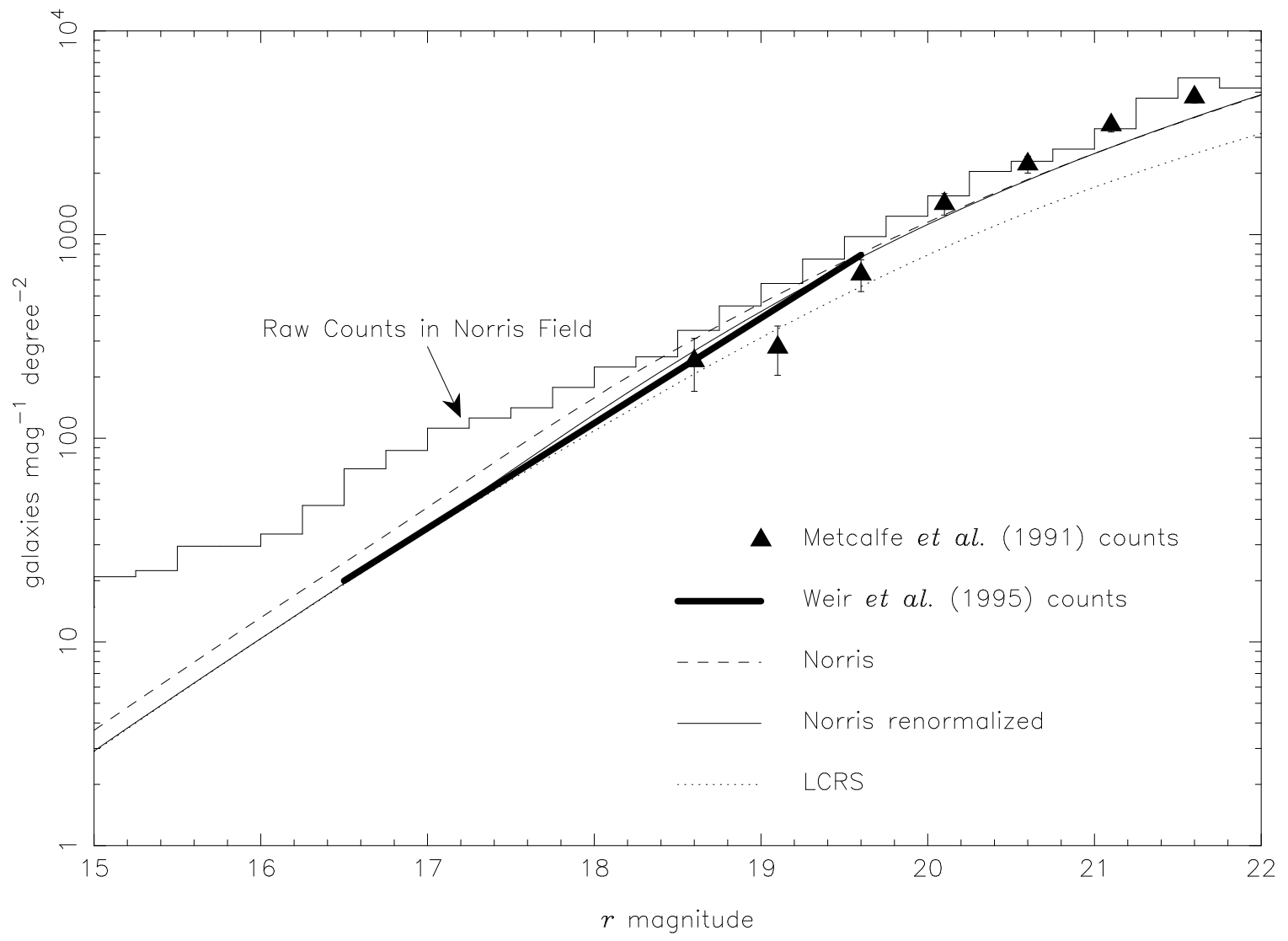

Fig. 6. - r-band differential galaxy counts. The counts in POSS-II field covering Corona Borealis, the POSS-II counts of Weir et al. (1995), and the CCD counts of Metcalfe et al. (1991) are given by the histogram, the thick solid line, and the triangles, respectively. We also plot the predicted differential number counts, computed using Equation 12, for three different LFs. The dashed line is based on the local LF normalized to the counts in the Corona Borealis field with the supercluster removed and on the LF computed for $0.2<z \leq 0.5$. Normalizing our local LF to the counts of Weir et al. (1995) for $16.5^{m} \leq r \leq 17.0^{m}$ and including the evolution of the LF for $z>0.2$, we obtain the predicted counts marked by the solid line, which agree well with the observed counts for $r \leq 20.0^{m}$. For comparison, we also plot the predicted counts based on the LCRS local LF and not including evolution of the LF beyond $z=0.2$. 


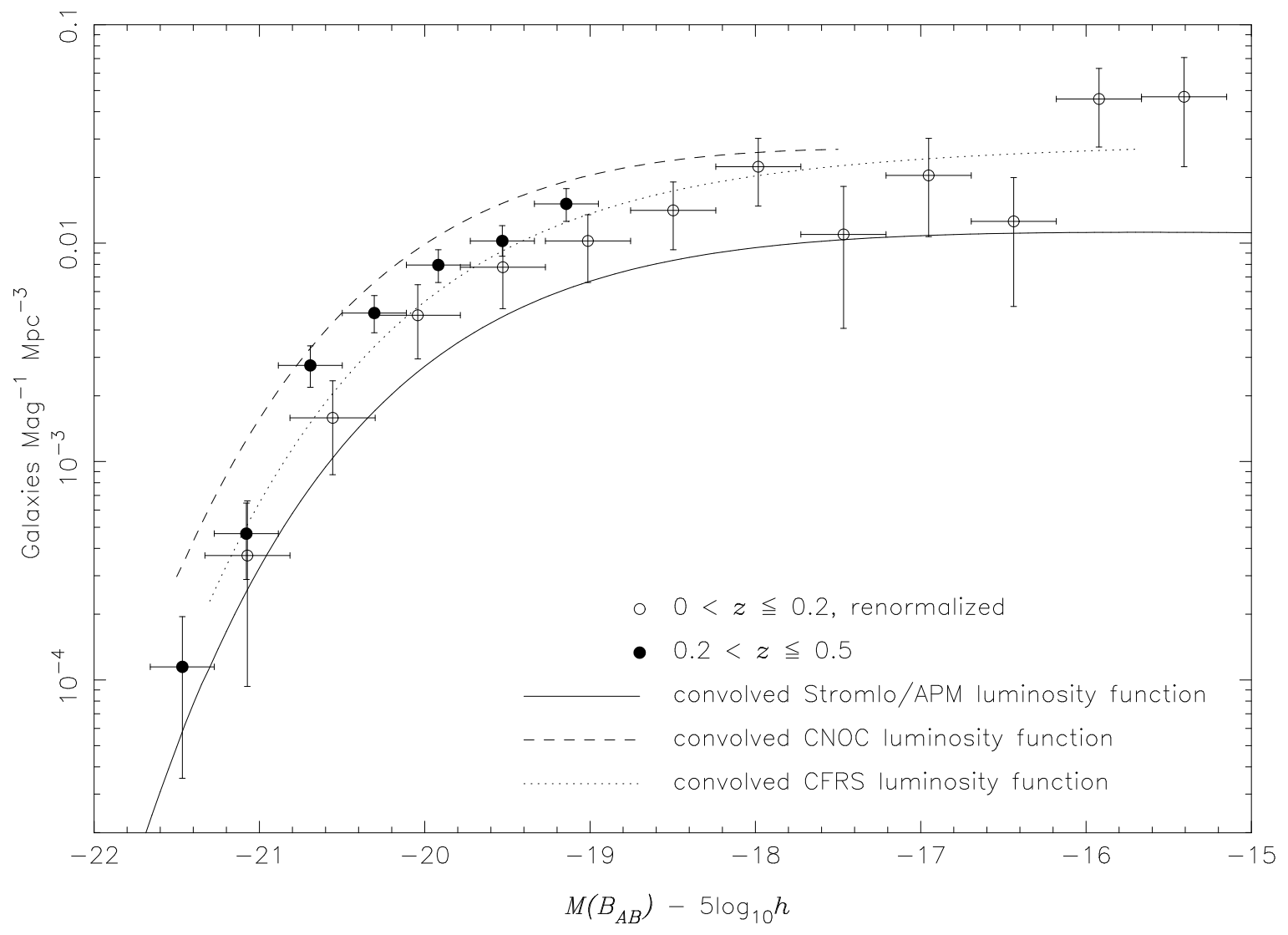

Fig. 7. - LFs for galaxies between $z=0$ and $z=0.5$. The filled circles show the LF for $0.2<z \leq 0.5$, while the unfilled circles show the LF for $0<z \leq 0.2$ normalized to the Weir et al. (1995) counts. The solid line is the convolved Schechter function fit to the local Stromlo/APM LF. The dashed and dotted lines give the convolved Schechter function fits to the LFs for $0.2<z<0.6$ and $0.2<z<0.5$ for CNOC and CFRS, respectively. Evolution of the LF is apparent. 


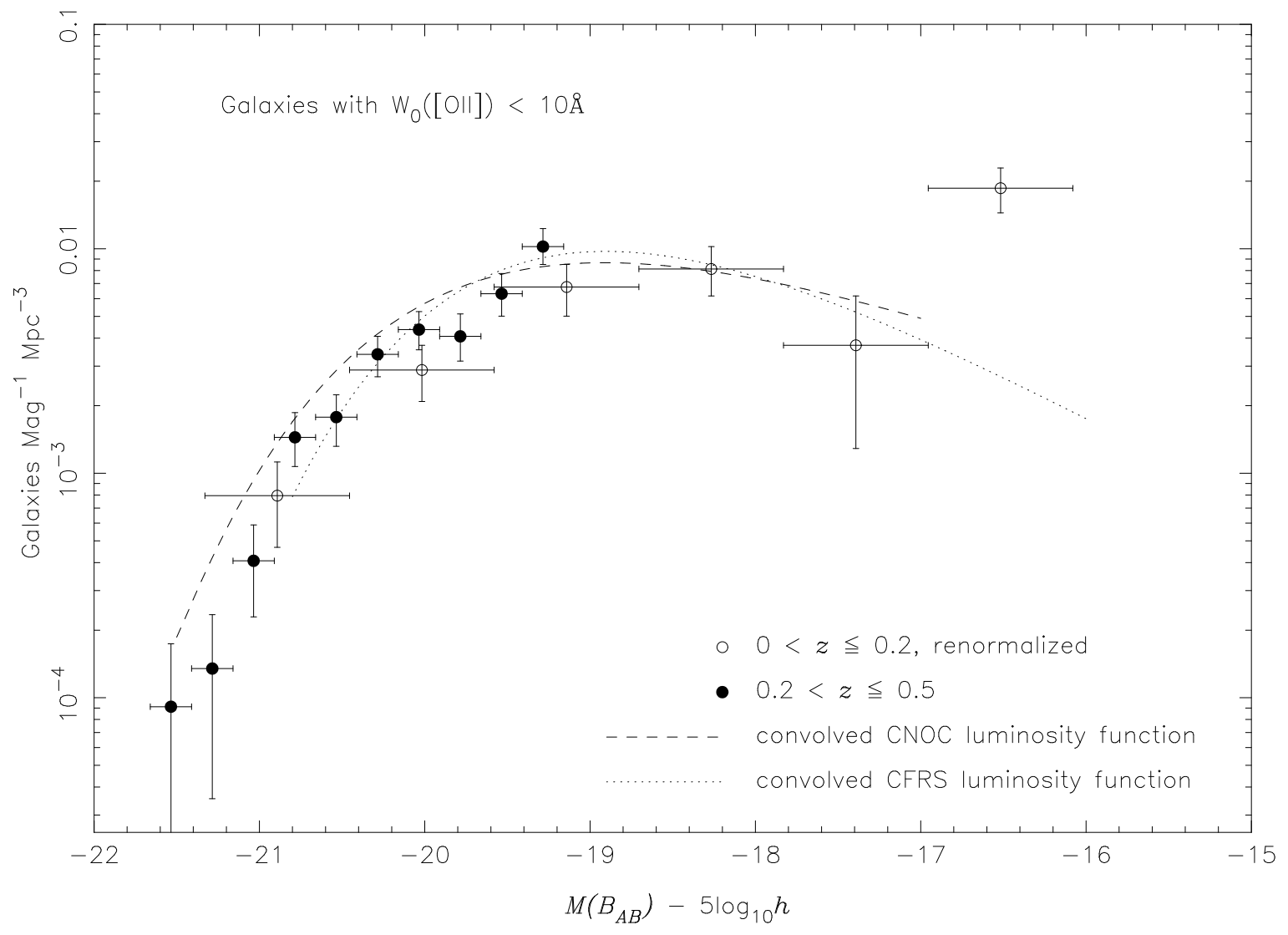

Fig. 8. - LF for galaxies with $W_{0}([\mathrm{O}$ II $])<10 \AA$ between $z=0$ and $z=0.5$. The filled circles are the LF of quiescent galaxies for $0.2<z \leq 0.5$; the unfilled circles are the local LF for quiescent galaxies with the normalization reduced by $21 \%$. The dashed and dotted lines show the LF of corresponding color-selected galaxies (i.e., colors redder than those of a CCW Sbc galaxy) in the redshift intervals $0.2<z<0.6$ and $0.2<z<0.5$ from CNOC and CFRS, respectively. There is no sign of evolution of the population of non-star-forming galaxies from $z=0$ to $z=0.5$. 


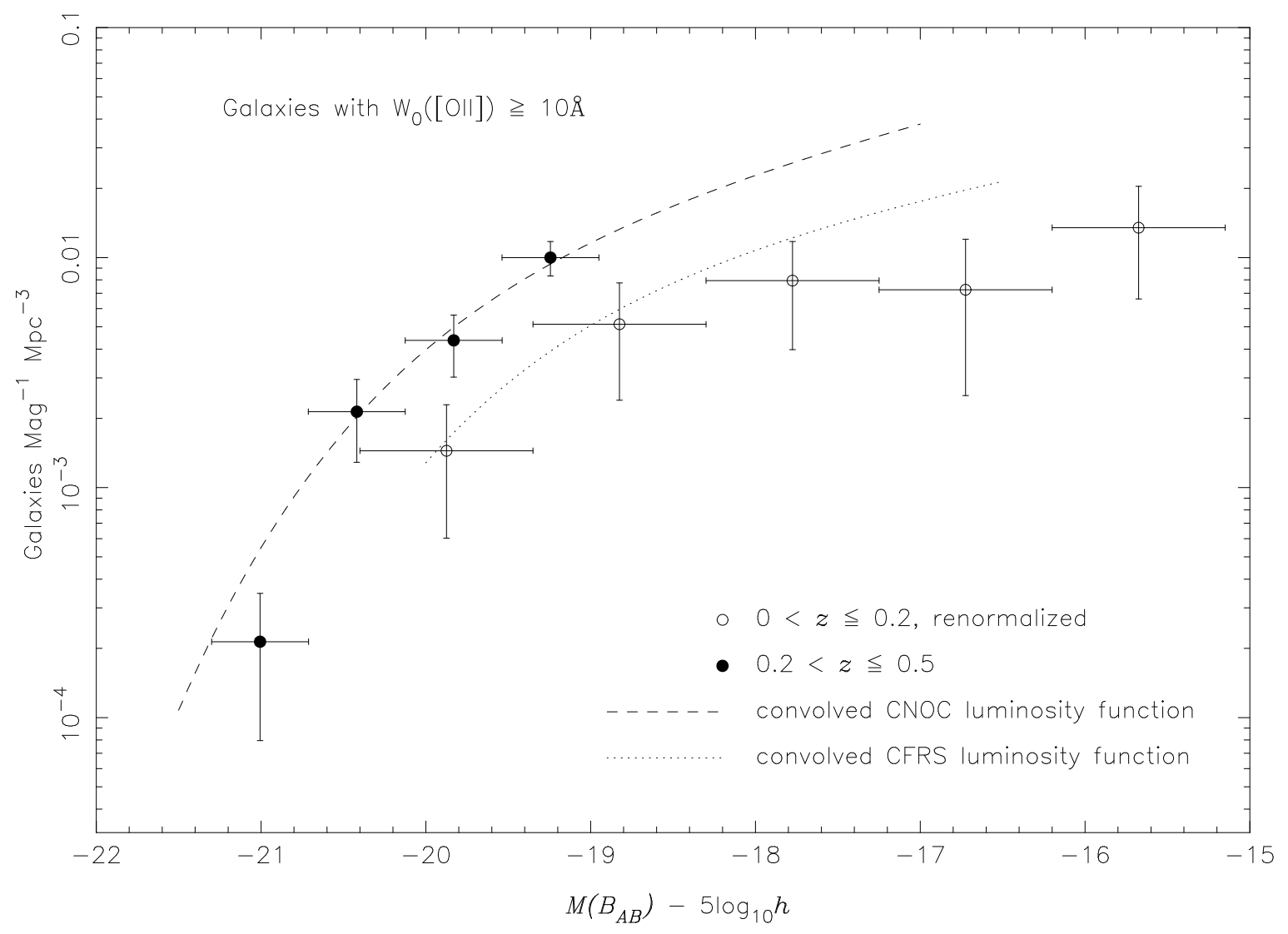

Fig. 9. - LF for galaxies with $W_{0}([\mathrm{O}$ II $])>10 \AA$ between $z=0$ and $z=0.5$. The filled circles are the LF of star-forming galaxies for $0.2<z \leq 0.5$; the unfilled circles are the local luminosity function for star-forming galaxies with the normalization reduced by $21 \%$. The dashed and dotted lines show the LF of corresponding color-selected galaxies (i.e., colors bluer than those of a CCW Sbc galaxy) in the redshift intervals $0.2<z<0.6$ and $0.2<z<0.5$ from CNOC and CFRS, respectively. The population of star-forming galaxies has evolved rapidly from $z=0$ to $z=0.5$. 


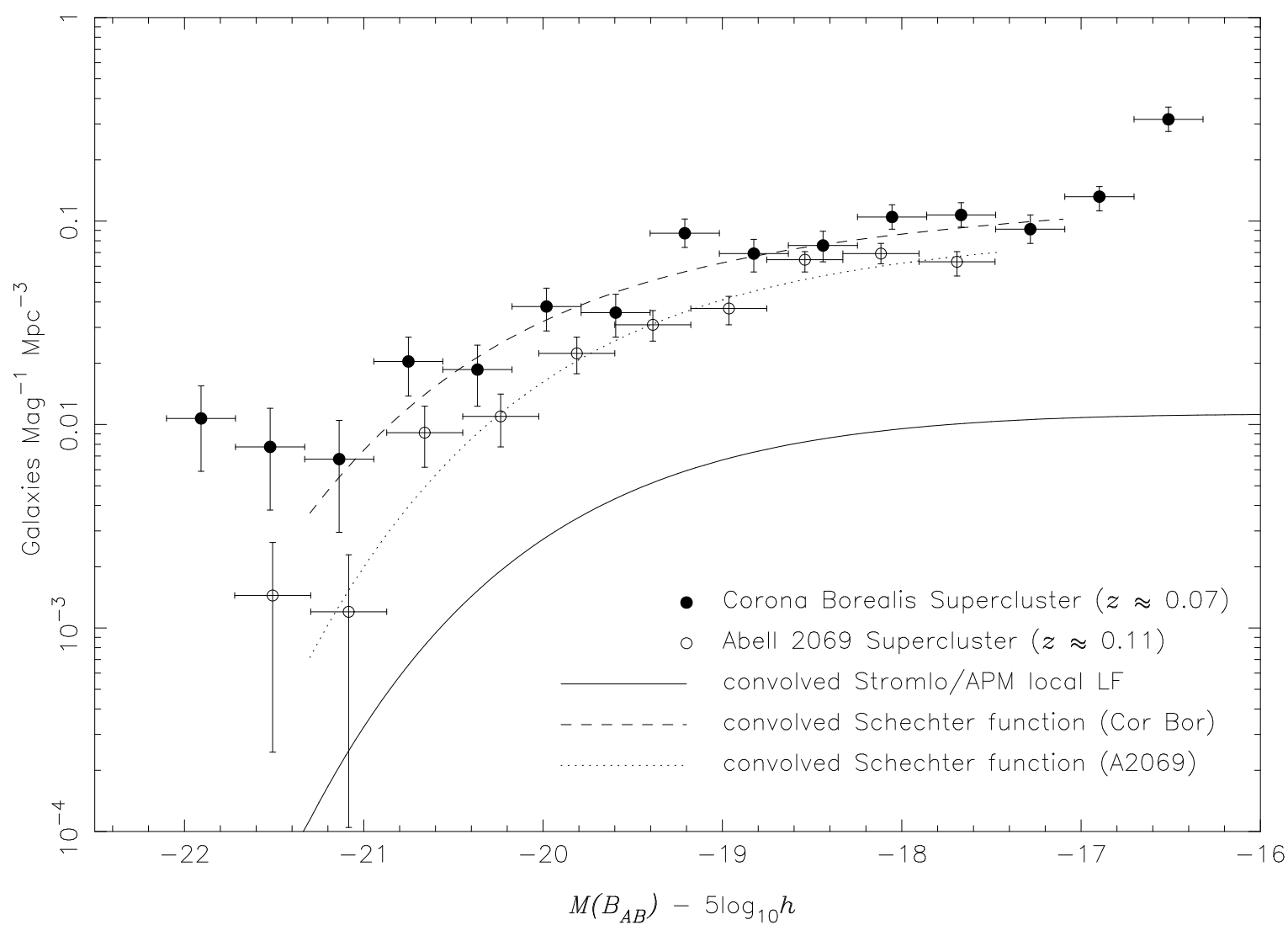

Fig. 10.- LFs of the Corona Borealis supercluster (filled circles) and the background A2069 supercluster (unfilled circles). The convolved Schechter function fits to the two supercluster luminosity functions $\left(M\left(B_{A B}\right)>-21.3^{m}\right)$ are given by the dashed (Corona Borealis) and dotted (A2069) lines. Brighter than $M\left(B_{A B}\right) \sim-21^{m}$, the superclusters have excess galaxies relative to the Schechter function fit. In addition, there is a hint that the LF of the Corona Borealis supercluster has a sharp upturn fainter than $M\left(B_{A B}\right) \sim-17^{m}$. The local LF from the Stromlo/APM survey is also plotted for comparison. 


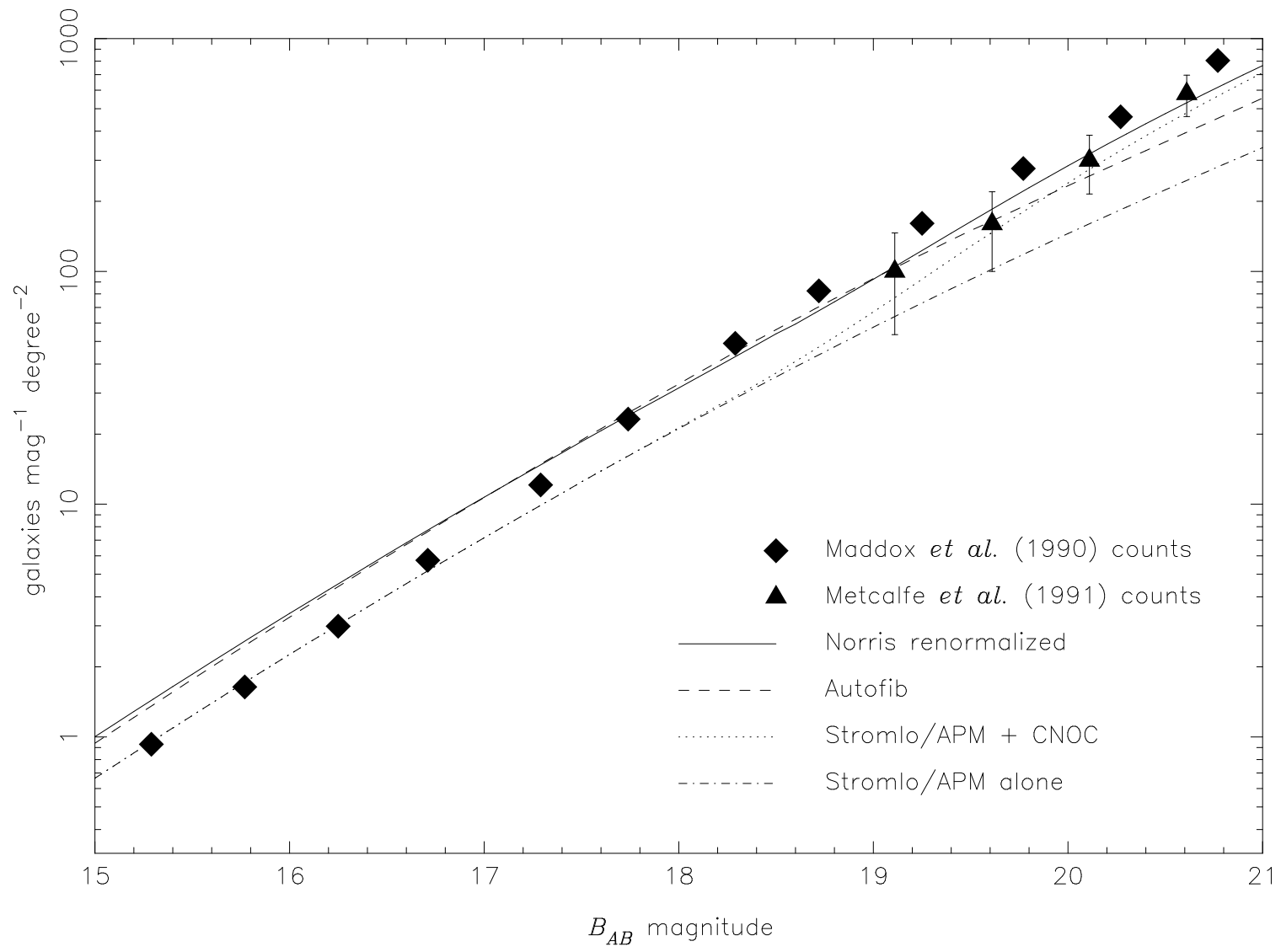

Fig. 11. - Observed and predicted differential counts in the $B_{A B}$ band. The diamonds are APM counts from Maddox et al. (1990), while the triangles are CCD-based counts from Metcalfe et al. (1991). We plot the predicted counts, using Equation 12, for several different observed LFs. The solid line gives the predicted counts for the evolving luminosity function measured in our survey (with the local LF normalized to the $r$-band counts). Similarly, the dashed line is the prediction from the Autofib survey, the dotted line the prediction using the Stromlo/APM local LF and the CNOC LF for $z>0.2$, and the dot-dashed line the prediction using just the Stromlo/APM LF alone. Our predictions and those based on the Autofib LF match the counts well for $18^{m} \lesssim B_{A B} \lesssim 21^{m}$, whereas the prediction which incorporates the Stromlo/APM local LF and the CNOC LF for $z>0.2$ matches the counts for $B_{A B} \lesssim 17^{m}$ and for $B_{A B} \gtrsim 19^{m}$, where most of the galaxies are at $z>0.2$. 


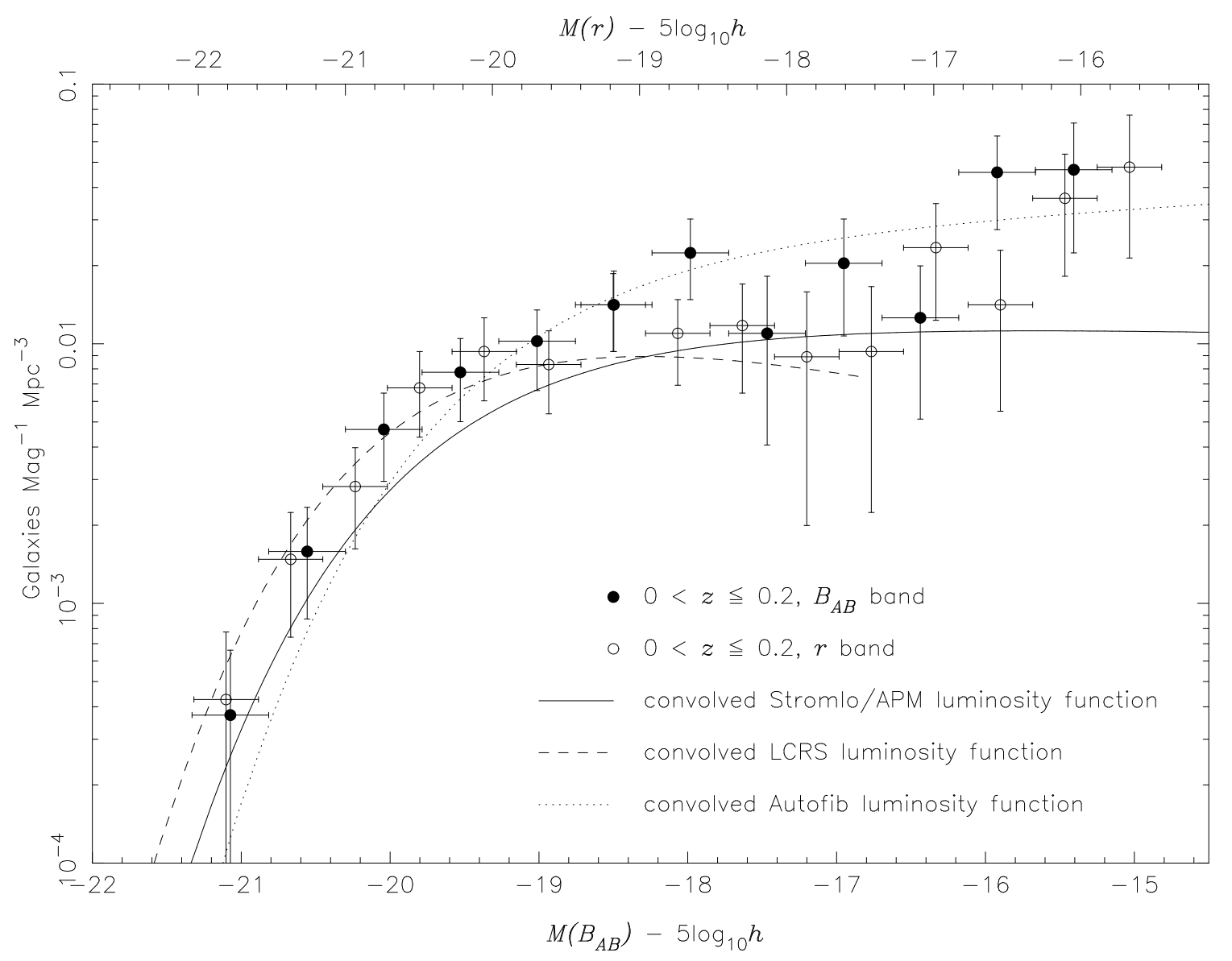

Fig. 12.- Assorted $B_{A B^{-}}$and $r$-band local LFs. The $B_{A B}$-band local LFs are plotted with respect to the bottom axis, while the $r$-band local LFs are plotted with respect to the top axis. The two axes are offset by $B_{A B}-r=0.72^{m}$, which is the median color we measure for field galaxies with $z<0.2$. The filled circles are the $B_{A B}$-band local LF, and the unfilled circles are the $r$-band local LF. The normalizations of both of our local LFs have been reduced by $21 \%$. The $B_{A B}$-band local LFs from the Stromlo/APM survey and the Autofib survey are plotted with the solid and dotted lines, respectively. The dashed line represents the local LF from the LCRS survey. After applying the color offset of $B_{A B}-r=0.72^{m}$, our $B_{A B^{-}}$and $r$-band local LFs agree well. However, in contrast to Lin et al. (1996b), we do not conclude that the LCRS $r$-band local LF matches the Stromlo/APM LF. 
$\mathrm{CB}-14.6497, g=20.9, g-r=1.6, z=0.433$

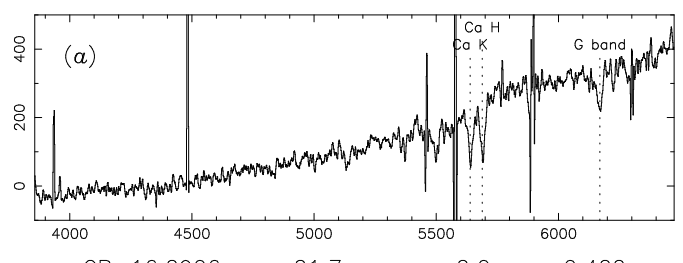

$\mathrm{CB}-16.2906, g=21.7, g-r=2.0, z=0.422$
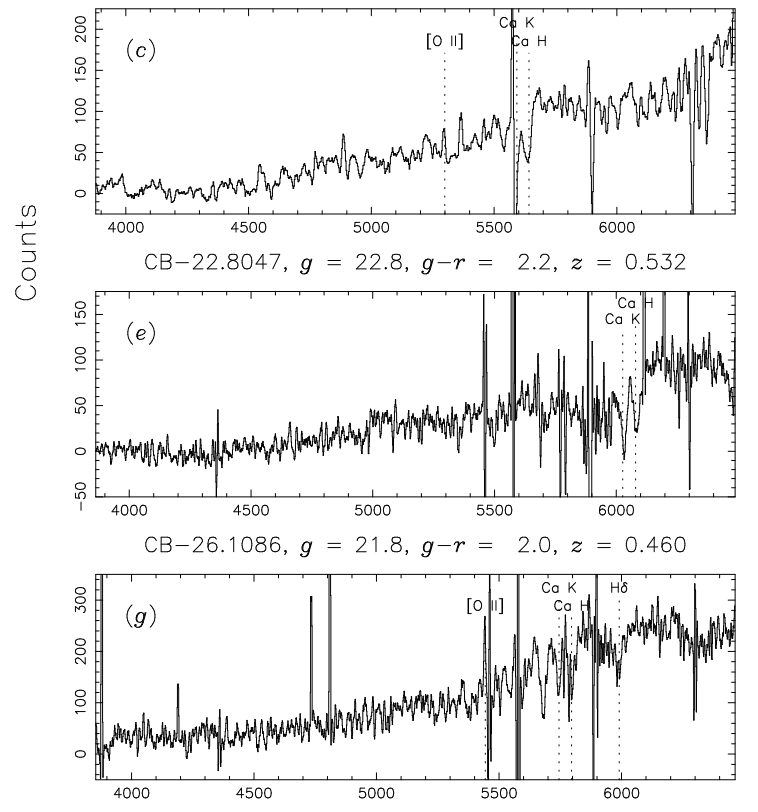

$\mathrm{CB}-14.7918, g=21.8, g-r=2.2, z=0.408$
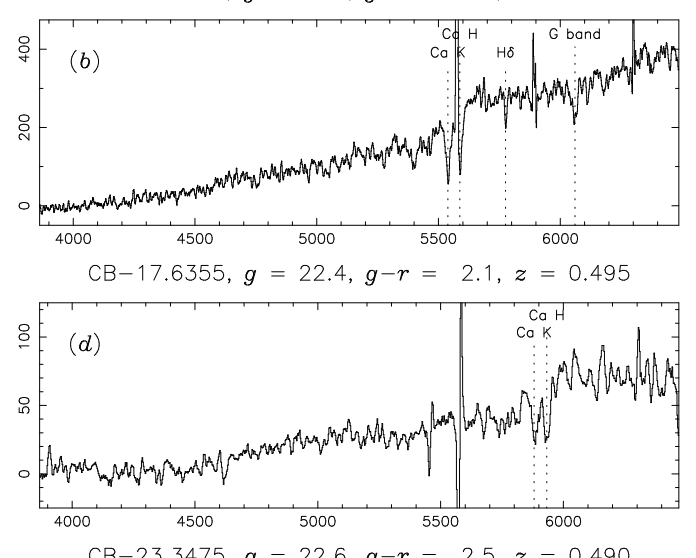

$\mathrm{CB}-23.3475, g=22.6, g-r=2.5, z=0.490$
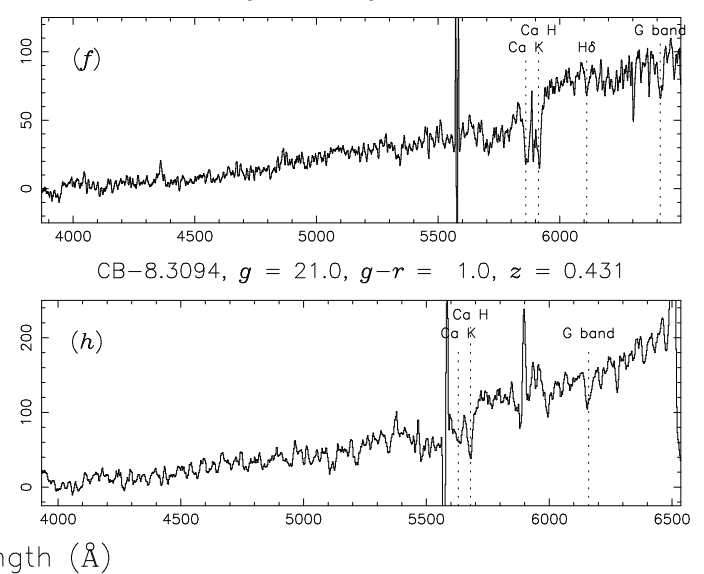

Fig. 13.- A representative sample of absorption-line galaxies at $0.4<z<0.6$ illustrating the strength of $\mathrm{Ca} \mathrm{H}, \mathrm{Ca} \mathrm{K}$, and the $4000 \AA$ break. 


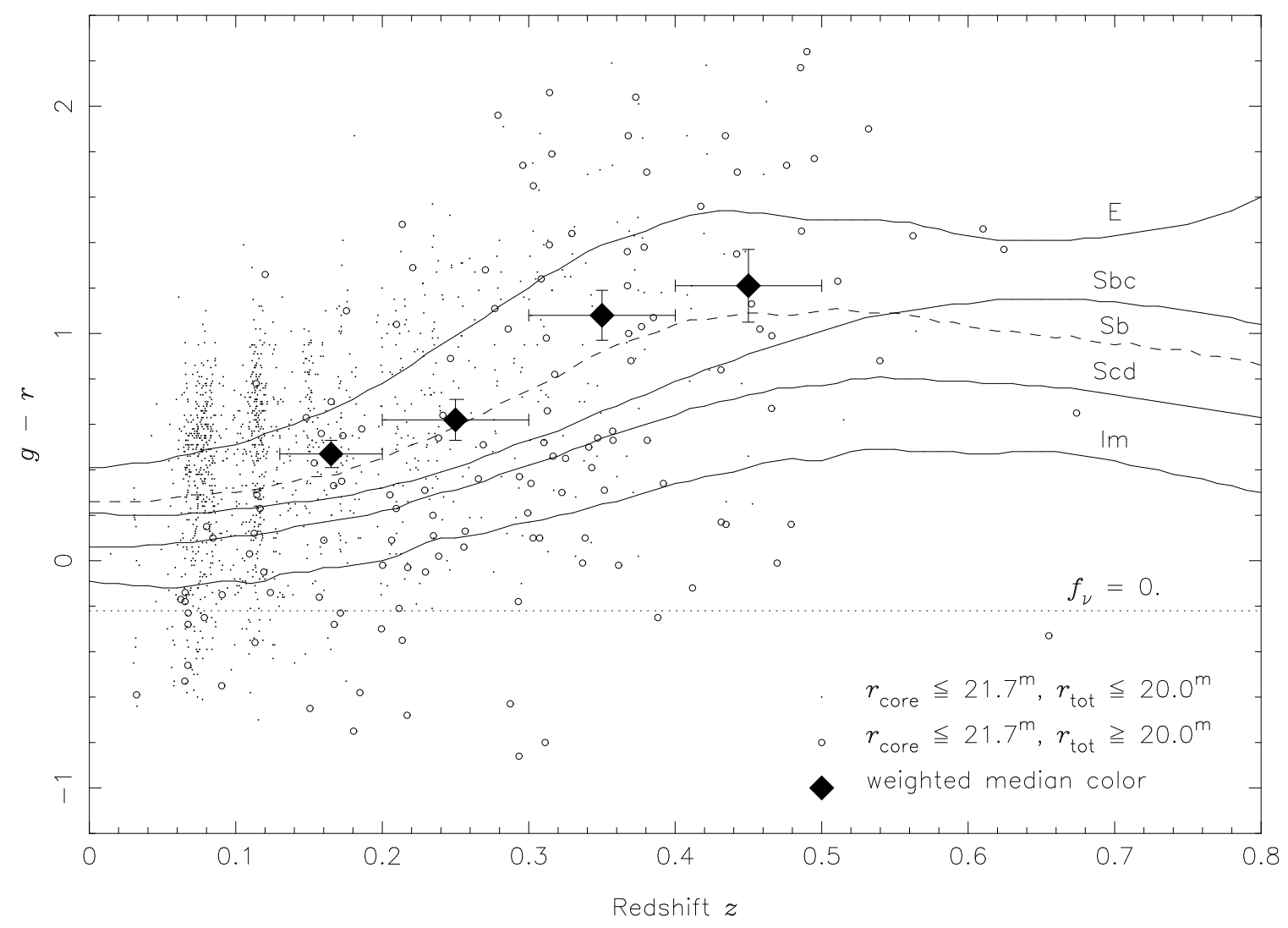

Fig. 14.- Median observed $g-r$ color as a function of redshift. We plot the observed $g-r$ color as a function of redshift along with the tracks of six representative model spectra. The bluest track is that of flat-spectrum galaxy, $f_{\nu}=0$. The other five tracks are those typical of the Hubble types E, Sb, Sbc, Scd, and Im. The large, filled triangles mark the weighted median $g-r$ color in the redshift intervals $0.13<z<0.2,0.2<z<0.3,0.3<z<0.4$, and $0.4<z<0.5$. The Sb track (the dotted line, from Bruzual \& Charlot 1993) matches the observed median galaxy colors to $z \sim 0.5$. 


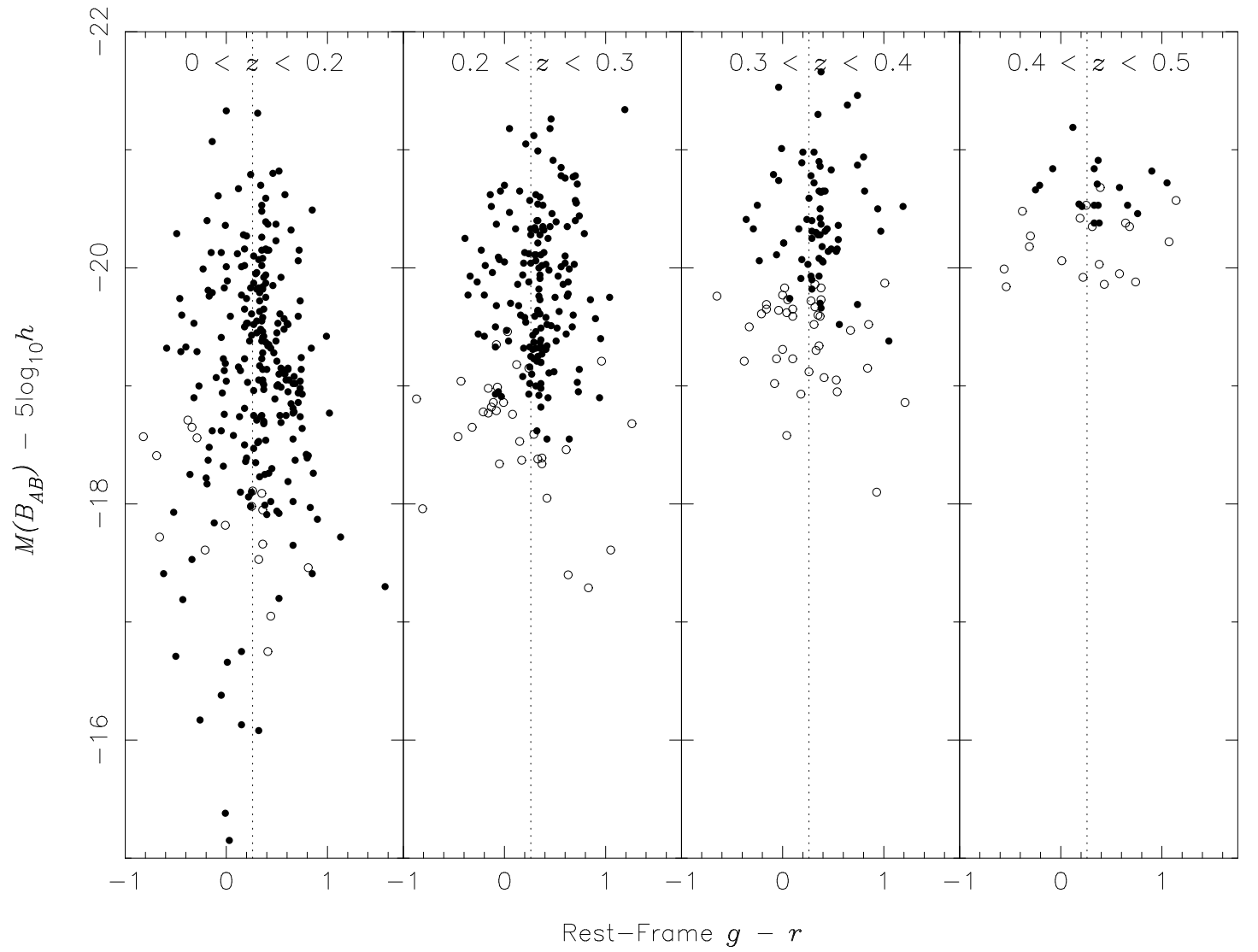

Fig. 15.- Absolute $B_{A B}$-band magnitude versus rest-frame $g-r$ colors for our survey galaxies, divided into four redshift intervals, $0<z<0.2$ (with the superclusters removed), $0.2<z<0.3,0.3<z<0.4$, and $0.4<z<0.5$. Galaxies with $r \leq 20.0^{m}$ are denoted by filled circles, and those with $r>20.0^{m}$ with unfilled circles. For $z<0.2$, the well-known color-magnitude relationship, i.e., that brighter galaxies are redder, is evident. However, at higher redshifts, the color-magnitude relationship disappears as the population of blue galaxies evolves and becomes intrinsically brighter. 


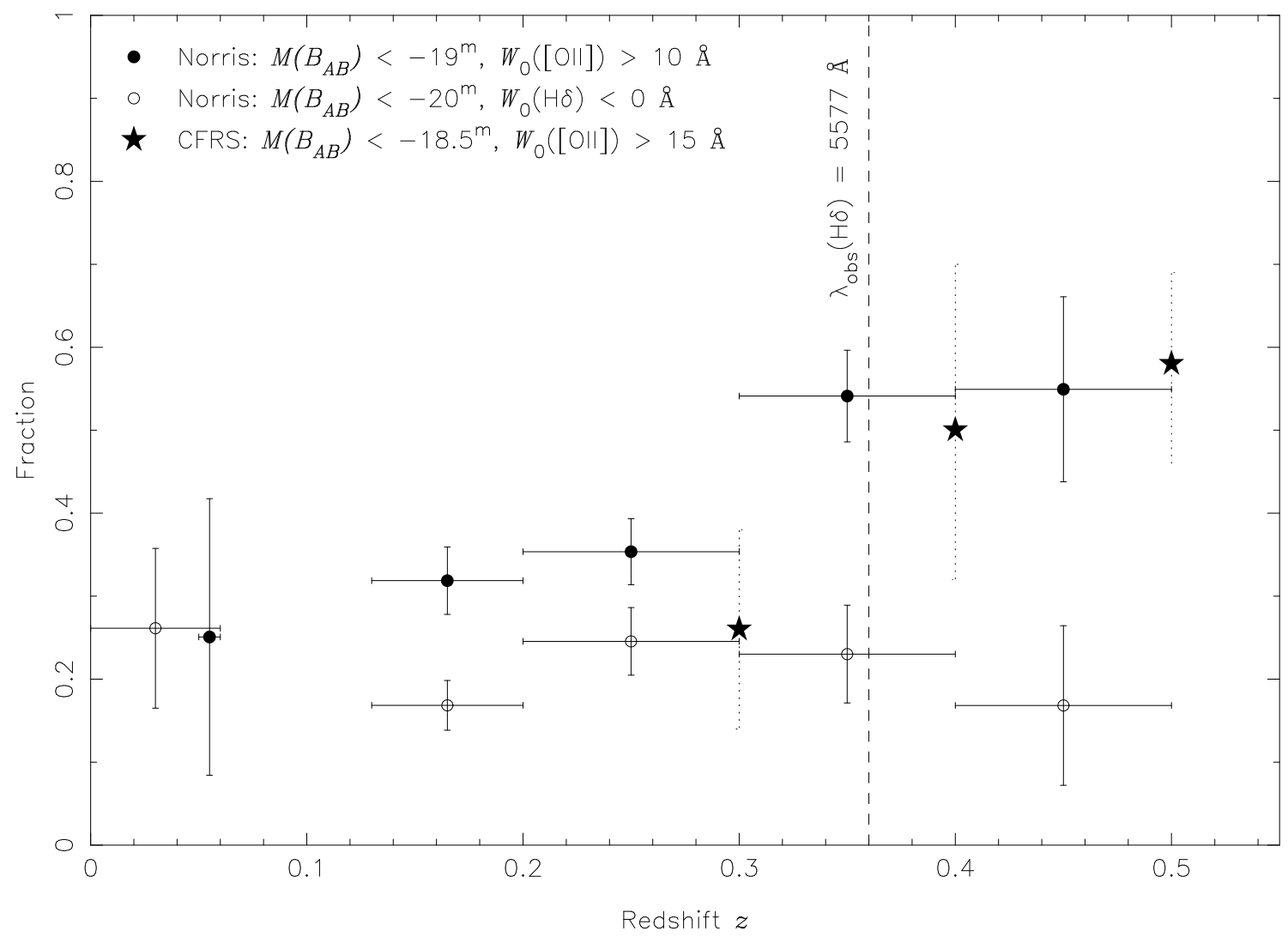

Fig. 16. - Fraction of galaxies with [O II] $\lambda 3727$ emission (filled circles) and $\mathrm{H} \delta \lambda 4101$ absorption (unfilled circles) as a function of redshift. The superclusters are excluded from the analysis. The dashed line indicates the redshift at which the observed wavelength of $\mathrm{H} \delta$ is $5577 \AA$; redward of this wavelength the increasingly bright sky makes measurement of the strength of $\mathrm{H} \delta$ more uncertain. The filled stars give the fraction of galaxies in the CFRS with $M\left(B_{A B}\right)<-18.5^{m}$ and $[\mathrm{O} \mathrm{II}]>15 \AA$ (Hammer et al. 1996). As [O II] is an indicator of ongoing star formation, the increasing fraction of galaxies exhibiting [O II] with redshift is evidence for an increase in the rate of star formation with redshift. However, the fraction of galaxies with $\mathrm{H} \delta$ absorption does not change with redshift, which argues against the star-formation activity in intermediate-redshift galaxies occurring predominantly in discrete bursts. 


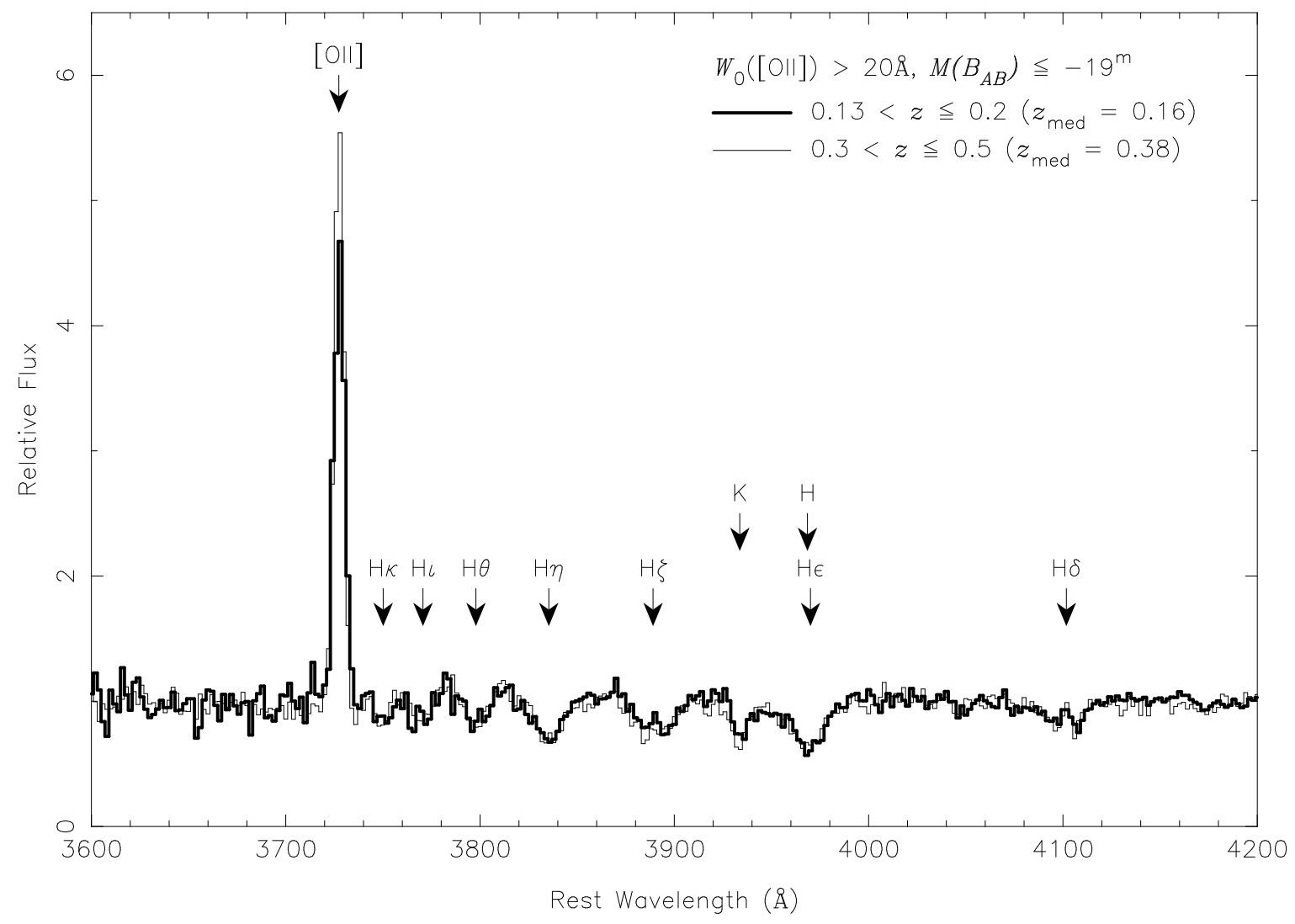

Fig. 17. - Composite spectra of galaxies with $W_{0}[\mathrm{O}$ II $]>20 \AA$ and $M_{B_{A B}} \leq-19^{m}$ for two redshift ranges, $0.13<z \leq 0.2$ (thick line) and $0.3<z \leq 0.5$ (thin line). The high redshift composite spectrum has stronger [O II], but the spectra are otherwise identical. In particular, the strength of $\mathrm{H} \delta$ has not changed with redshift. 


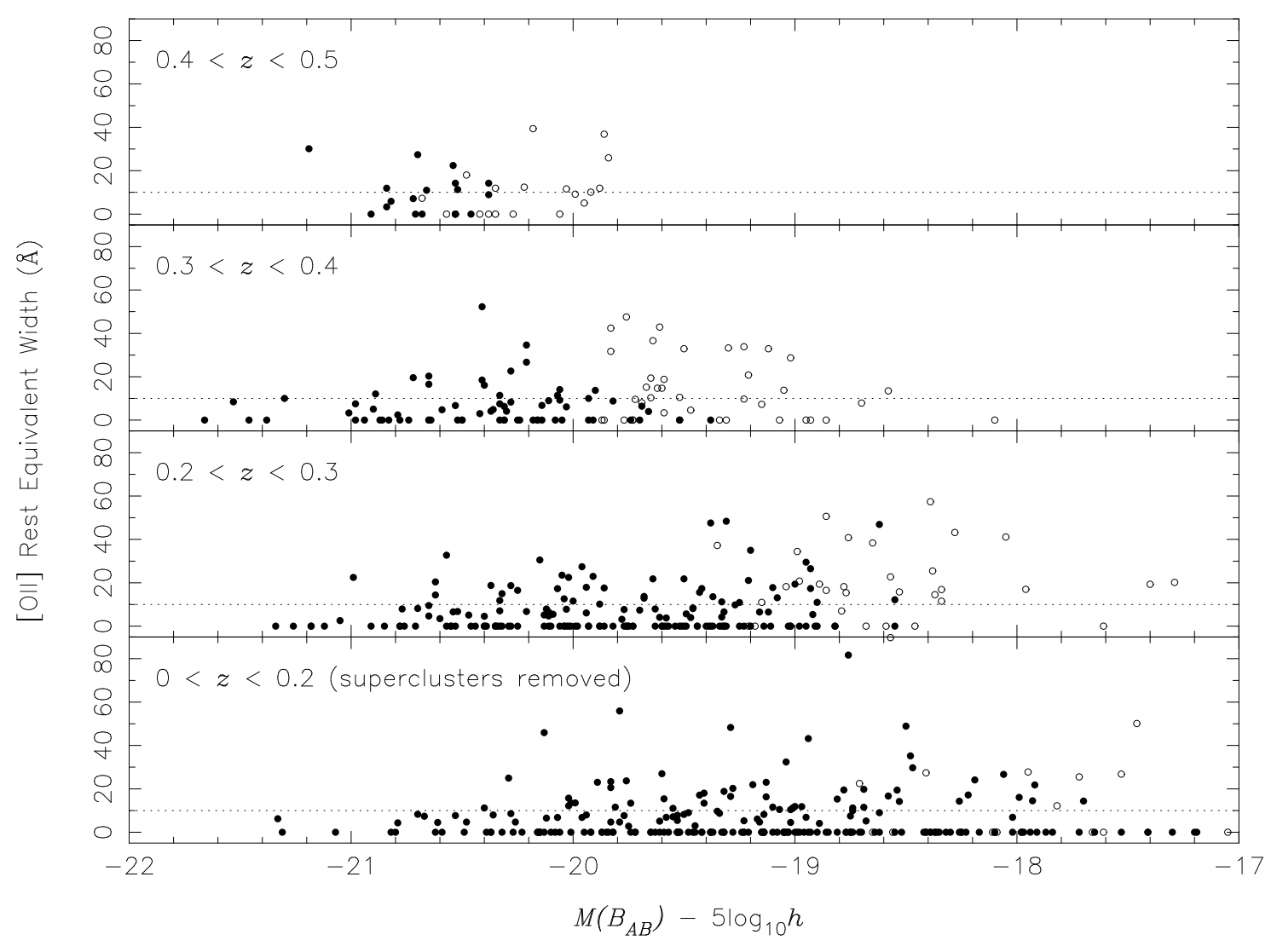

Fig. 18. - Rest equivalent widths of [O II] as a function of absolute $B_{A B}$-band magnitude in four redshift intervals, $0<z<0.2$ (with the superclusters removed), $0.2<z<0.3$, $0.3<z<0.4$, and $0.4<z<0.6$. The filled circles have $r \leq 20^{m}$, and the unfilled circles have $r>20^{m}$. The dotted line marks an [O II] equivalent width of $10 \AA$. This figure illustrates two important points. The range of star formation rates, as measured by the [O II] equivalent widths, at $z \sim 0.5$ is similar to that at low redshift. However, the luminosities of galaxies undergoing rapid star formation increases progressively from low redshift, where ongoing star formation is mainly restricted to sub- $L^{*}$ galaxies, to high redshift, where even some super- $L^{*}$ galaxies are rapidly forming stars. 